Article

\title{
Genome-Wide Identification, Evolution, and Comparative Analysis of B-Box Genes in Brassica rapa, B. oleracea, and B. napus and Their Expression Profiling in B. rapa in Response to Multiple Hormones and Abiotic Stresses
}

\author{
Sonam Singh ${ }^{1,+}$, Sushil Satish Chhapekar ${ }^{1,+}{ }^{\circledR}$, Yinbo Ma ${ }^{1}{ }^{10}$, Jana Jeevan Rameneni ${ }^{1}$, Sang Heon $\mathrm{Oh}^{1}$, \\ Jusang Kim ${ }^{2}$, Yong Pyo Lim ${ }^{1, *}$ and Su Ryun Choi ${ }^{1, *}$ \\ 1 Department of Horticulture, College of Agriculture and Life Science, Chungnam National University, \\ Daejeon 34134, Korea; sonamsingh688@gmail.com (S.S.); sushilchhapekar@gmail.com (S.S.C.); \\ mayinbo@126.com (Y.M.); saijeevan7@gmail.com (J.J.R.); rederaser@naver.com (S.H.O.) \\ 2 Breeding Research Institute, Dayi International Seed Co., Ltd., 16-35 Ssiat-gil, Baeksan-myeon, \\ Gimje 54324, Jeollabuk-do, Korea; juzu1227@dayiseed.co.kr \\ * Correspondence: yplim@cnu.ac.kr (Y.P.L.); srchoi@cnu.ac.kr (S.R.C.); Tel.: +82-42-821-8846 (Y.P.L. \& S.R.C.); \\ Fax: +82-42-821-8847 (Y.P.L. \& S.R.C.) \\ + These authors contributed equally and shares first authorship.
}

Citation: Singh, S.; Chhapekar, S.S.; Ma, Y.; Rameneni, J.J.; Oh, S.H.; Kim, J.; Lim, Y.P.; Choi, S.R. Genome-Wide Identification, Evolution, and Comparative Analysis of B-Box Genes in Brassica rapa, B. oleracea, and B. napus and Their Expression Profiling in B. rapa in Response to Multiple Hormones and Abiotic Stresses. Int. J. Mol. Sci. 2021, 22, 10367. https:// doi.org/10.3390/ijms221910367

Academic Editor: Marcello Iriti

Received: 24 July 2021

Accepted: 22 September 2021

Published: 26 September 2021

Publisher's Note: MDPI stays neutral with regard to jurisdictional claims in published maps and institutional affiliations.

Copyright: (c) 2021 by the authors. Licensee MDPI, Basel, Switzerland. This article is an open access article distributed under the terms and conditions of the Creative Commons Attribution (CC BY) license (https:// creativecommons.org/licenses/by/ $4.0 /)$.

\begin{abstract}
The B-box zinc-finger transcription factors are important for plant growth, development, and various physiological processes such as photomorphogenesis, light signaling, and flowering, as well as for several biotic and abiotic stress responses. However, there is relatively little information available regarding Brassica B-box genes and their expression. In this study, we identified 51, 52, and 101 non-redundant genes encoding B-box proteins in Brassica rapa (BrBBX genes), B. oleracea (BoBBX genes), and $B$. napus (BnBBX genes), respectively. A whole-genome identification, characterization, and evolutionary analysis (synteny and orthology) of the B-box gene families in the diploid species B. rapa (A genome) and B. oleracea (C genome) and in the allotetraploid species B. napus (AC genome) revealed segmental duplications were the major contributors to the expansion of the Brassica $B B X$ gene families. The Brassica BBX genes were classified into five subgroups according to phylogenetic relationships, gene structures, and conserved domains. Light-responsive cis-regulatory elements were detected in many of the $B B X$ gene promoters. Additionally, BrBBX expression profiles in different tissues and in response to various abiotic stresses (heat, cold, salt, and drought) or hormones (abscisic acid, methyl jasmonate, and gibberellic acid) were analyzed by qRT-PCR. The data indicated that many B-box genes (e.g., BrBBX13, BrBBX15, and BrBBX17) may contribute to plant development and growth as well as abiotic stress tolerance. Overall, the identified BBX genes may be useful as functional genetic markers for multiple stress responses and plant developmental processes.
\end{abstract}

Keywords: B-box; BBX; Brassica rapa; Brassicaceae; evolution; gene duplication; synteny; abiotic stress; hormonal stress

\section{Introduction}

The B-box $(B B X)$ genes have recently been revealed to encode multifarious zincfinger transcription factors with diverse functions in several plant species [1,2]. The $B B X$ transcription factor family members are major contributors to several activities, including the photoperiod-related regulation of flowering, plant developmental processes, seedling photomorphogenesis, seed germination, and abiotic and biotic stress responses [3]. Plant $B B X$ transcription factors have one or two $\mathrm{N}$-terminal $\mathrm{BBX}$ domains with or without a C-terminal CCT domain (COL-like/CO or TOC1 motif), unlike the corresponding proteins in animals, which include a RING finger and a coiled-coil domain along with the BBX domain. 
In plants, based on the distance between the zinc-binding residues and their consensus sequence, the BBX domains can be divided into the B-box1 (B1) and B-box2 (B2) classes [4]. The CCT domain, which is highly conserved and composed of 42 or 43 amino acids, is important for nuclear protein transport and the regulation of transcription [5,6]. The BBX domain is predicted to include conserved histidine $(\mathrm{H})$, cysteine $(\mathrm{C})$, and aspartic acid (D) residues that modulate protein-protein interactions [4,7,8]. Khanna et al. identified 32 BBX genes in Arabidopsis thaliana and named them AtBBX1-32 [4]. These 32 BBX genes were further classified into Groups I-V depending on the number of BBX and CCT domains [3,4,9] (Figure 1). In A. thaliana, CONSTANS (CO)/AtBBX1, which was the first member of the BBX family to be studied, is the main coordinator of flowering time because it induces FLOWERING LOCUS T (FT) expression $[9,10]$.

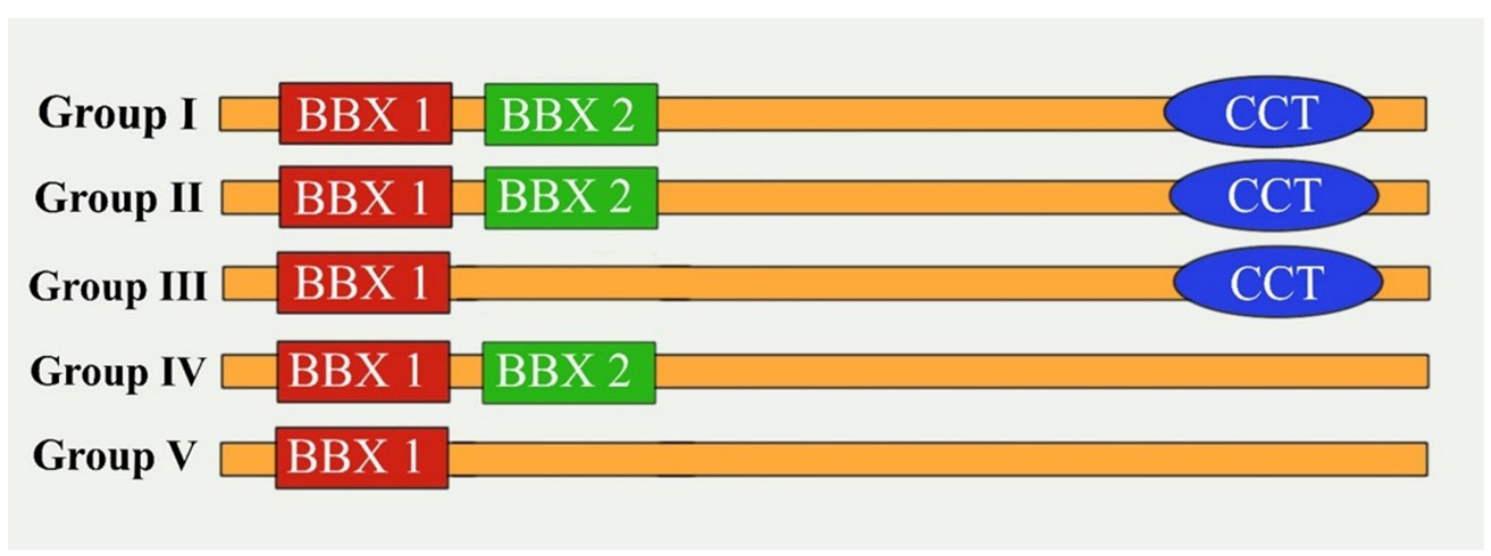

Figure 1. Scheme illustrating major features of BBX proteins in each structure group (Modified from Talar et al. [11]).

In transgenic $A$. thaliana plants, the overexpression of the $C O$ gene results in abnormal flowering under short-day and long-day conditions, whereas a mutation of this gene delays flowering under long-day conditions [12-14]. Approximately $10 B B X$ genes have been identified as crucial regulators of early photomorphogenesis, including $B B X 4, B B X 7$, $B B X 21$ and $B B X 32$, which control the flowering time [15-17]. Previous studies on $A$. thaliana revealed that $B B X 19, B B X 20, B B X 24, B B X 25, B B X 28$, and $B B X 32$ negatively regulate photomorphogenesis [18-20], whereas $B B X 4, B B X 21, B B X 22$ and $B B X 23$ have the opposite effect [21-23]. Various reports confirmed that BBX proteins associate with ELONGATED HYPOCOTYL 5 (HY5) at the protein or gene level and modulate plant photomorphogenesis. For example, in response to irradiation, BBX21 binds to BBX22 and HY5 as well as its own promoter and activates transcription, whereas $B B X 11$ binds to the $H Y 5$ promoter to modulate expression [21,22,24,25]. Intriguingly, in A. thaliana exposed to red light, $B B X 4$ accumulates and interacts with $p h y B$, thereby enhancing photomorphogenesis [26]. A similar mechanism was also detected in other plant species. Specifically, in apple, MdHY5 promoter activity is inhibited by MdBBX37. Additionally, MdHY5 expression is modulated by $M d B B X 25 / M d C O L 4$ and $M d B B X 22$, which binds directly to the promoter and decreases or increases transcription $[27,28]$. In rice, OsHY5L1 expression is induced by OsBBX14 to positively affect photomorphogenesis [29]. In tomato, SIBBX20 interacts with the PSY1 promoter, which enhances carotenoid biosynthesis [30].

BBX proteins might also have an important role in abiotic stress responses and hormone signaling networks. In tomato, rice, apple, and grapevine, some stress- and hormone signaling-related cis-elements, such as the LTR, MBS, HSE, ABA-responsive element (ABRE), ethylene-responsive element (ERE), and gibberellin-responsive element (GARE), have been detected in the promoter region of most $B B X$ genes, implying these genes are involved in hormone signaling pathways and abiotic stress responses [8,31-33] However, their actual functions related to abiotic stress responses and hormone signaling pathways remain unknown. The $A$. thaliana AtBBX24 gene encodes a salt tolerance protein (STO), 
which can increase the salt tolerance of transgenic yeast cells. Furthermore, the overexpression of AtBBX24 in A. thaliana leads to significantly increased salt tolerance and root growth $[34,35]$. In chrysanthemum, CmBBX24 delays flowering and increases cold and drought tolerance [9]. In $A$. thaliana, STO interacts with CLONE EIGHTY ONE/RADICALINDUCED CELL DEATH1 (CEO/RCD1), which negatively regulates the expression of a broad range of stress-related genes [36,37]. Another A. thaliana BBX family member, $A t B B X 18$, negatively regulates both heat tolerance and photomorphogenesis, whereas in grapevine, $V v B B X 32$ overexpression results in cold stress tolerance [38,39]. Moreover, $A t B B X 18$ downregulates the expression of other heat-responsive genes, such as Hsp70, DGD1, APX2, and Hsp101, which adversely affects seedling survival and germination under heat stress conditions [39]. Other studies demonstrated that $A t B B X 18$ functions as a positive regulator in the gibberellin (GA) signaling network, but $A t B B X 20$ is a negative regulator in the brassinosteroid signaling pathway $[18,40]$.

The genus Brassica comprises approximately 100 species, including important vegetable and oilseed crops cultivated worldwide. Chinese cabbage (Brassica rapa L.), broccoli (Brassica oleracea L.), and rapeseed (Brassica napus L.) are mostly cultivated as vegetables for human consumption and for producing oil, condiments, and fodder because of their nutrient contents, including vitamins, proteins, and minerals (e.g., zinc, iron, sodium, and potassium) [41-43].

To date, $B B X$ genes have been identified in numerous plant species, including rice [8], soybean [44], tomato [31], pear [45,46], potato [47], apple [32], Rosaceae [48], Poaceae [49], and grapevine [33]. Although there are several studies on BBX genes in many plants, they have not been comprehensively characterized in Brassica species and their evolution and functions related to abiotic and hormone stress responses have yet to be studied. Therefore, we identified $51 B B X$ genes in B. rapa, 52 BBX genes in B. oleracea, and $101 B B X$ genes in $B$. napus and subsequently analyzed their evolutionary relationships, structures, expression patterns, cis-elements, and chromosomal locations as well as their effects on various abiotic and hormone stress responses. The results of this study will provide new insights regarding the evolutionary importance of the functional divergence of these genes and will lay the foundation for future investigations on the BBX proteins in Brassicaceae.

\section{Results}

2.1. Identification of BBX Genes in Brassica Species and Physicochemical Analysis and Subcellular Localization of the Encoded Proteins

The Hidden Markov Model as well as the $A$. thaliana BBX gene sequences obtained from the TAIR database were used to identify the $B B X$ gene family members in $B$. rapa, B. oleracea, and B. napus. The BBX domains of the BBX genes in all three Brassica species were identified using the NCBI-CDD, SMART, and Pfam databases. A total of 51,52, and $101 B B X$ genes were identified in B. rapa, B. oleracea, and B. napus, respectively, after the redundant sequences were eliminated (Table 1). The 204 non-redundant $B B X$ genes were renamed as B. rapa BrBBX01-51 (Table S1), B. oleracea BoBBX01-52 (Table S2), and B. napus $B n B B X 01-101$ (Table S3) according to the relative linear order on each chromosome and widely used nomenclature. Furthermore, $B n B B X$ referred to the $B B X$ genes in the whole $B$. napus genome, whereas $B n A B B X$ and $B n C B B X$ genes belonged to the $A$ and $C$ genomes, respectively.

Table 1. Summary of B-box genes in B. rapa, B. oleracea, B. napus and Arabidopsis thaliana.

\begin{tabular}{ccccccc}
\hline \multicolumn{7}{c}{ B-Box } \\
\hline Species & Group I & Group II & Group III & Group IV & Group V & Total \\
\hline A. thaliana & 6 & 7 & 4 & 8 & 7 & 32 \\
B. rapa & 8 & 11 & 6 & 17 & 9 & 51 \\
B. oleracea & 9 & 11 & 7 & 16 & 9 & 52 \\
B. napus & 26 & 13 & 22 & 26 & 14 & 101 \\
\hline
\end{tabular}


The molecular weights of the Brassica $\mathrm{BBX}$ proteins ranged from $11.253 \mathrm{kDa}(\mathrm{BrBBX} 3)$ to $61.128 \mathrm{kDa}$ (BrBBX9) in B. rapa, from $12.397 \mathrm{kDa}$ (BoBBX3) to $46.391 \mathrm{kDa}$ (BoBBX23) in B. oleracea, and from $4.801 \mathrm{kDa}(\mathrm{BnABBX} 28)$ to $62.649 \mathrm{kDa}(\mathrm{BnCBBX} 69)$ in B. napus. The pI ranged from 11.60 (BnCBBX96) to 3.81 (BnABBX7). On the basis of their $\mathrm{pI}$, most $B$. rapa $\mathrm{BBX}$ proteins were acidic; the exceptions were BrBBX3, BrBBX8, BrBBX9, BrBBX13, BrBBX19, BrBBX24, and BrBBX51, which had a pI greater than 7. Regarding B. oleracea and B. napus, 15 and $30 \mathrm{BBX}$ proteins had a pI exceeding 7, respectively. However, most of the Brassica BBX proteins were unstable, with an instability index greater than 40 (Tables S1-S3). The Brassica BBX proteins lacked a transmembrane segment, with the exception of BrBBX33 and BnABBX3 (data not shown). The GRAVY index for almost all BBX proteins $(<0)$ reflected the hydrophilic nature of these proteins. Only BnABBX3 had a GRAVY index greater than 0 (0.098). The aliphatic index for all Brassica BBX proteins ranged from 46.04 to 85.73. The subcellular localization analysis revealed that the BrBBX proteins were mostly localized in the nucleus (30 BrBBXs), followed by the chloroplast (11 BrBBXs), cytoplasm (9 BrBBXs), and the endoplasmic reticulum (1 BrBBX). In B. oleracea, 31, 14, 6, and $1 \mathrm{BBX}$ proteins were localized in the nucleus, chloroplast, cytoplasm, and mitochondria, respectively. In B. napus, $62,22,12$, and 2 BBX proteins were localized in the nucleus, chloroplast, cytoplasm, and mitochondria, respectively, whereas one BBX was localized in the extracellular matrix, vacuole, and endoplasmic reticulum (Tables S1-S3). The most common amino acids in the Brassica BBX proteins were serine (S), alanine (A), leucine (L), and glutamic acid (L), whereas threonine $(\mathrm{T})$, cysteine $(\mathrm{C})$, and proline $(\mathrm{P})$ were the least abundant amino acids (Tables S1-S3).

\subsection{Analyses of the Phylogenetic Relationships, Conserved Domains, Structures, and Motifs of B. rapa, B. oleracea, and B. napus BBX Genes}

To elucidate the evolutionary relationships and functional divergence among Brassica $\mathrm{BBX}$ proteins, the sequences of $51 \mathrm{~B}$. rapa $\mathrm{BBX}$ proteins, 52 B. oleracea $\mathrm{BBX}$ proteins, 101 B. napus BBX proteins, and 32 A. thaliana BBX proteins were used to construct an unrooted phylogenetic tree (Figure 2). The BBX proteins were divided into five groups (Groups I-V) according to previous studies [3,4]. The Group I BBXs contained B1 and B2 domains along with the CCT domain; the Group II BBXs had the same domains as the Group I proteins, but the B2 domain differed at the amino acid level; the Group III BBXs included the B1 domain and the CCT domain; the Group IV BBXs had B1 and B2 domains, but lacked the CCT domain; and the Group V BBXs only had a single B1 domain. Groups I and IV had the most BBX members (Figure 2). Some of the BBXs were not categorized as expected. For example, $B r B B X 5, B r B B X 48, B o B B X 4$, and $B o B B X 45$ included only the B1 domain and the CCT domain, suggesting they should belong to Group III, but they were in Group II in the phylogenetic tree; likewise, BrBBX16 and BnCBBX96 should be in Group V as they included only the B1 domain, but they fall in Group IV and II, respectively (Figure 2). 


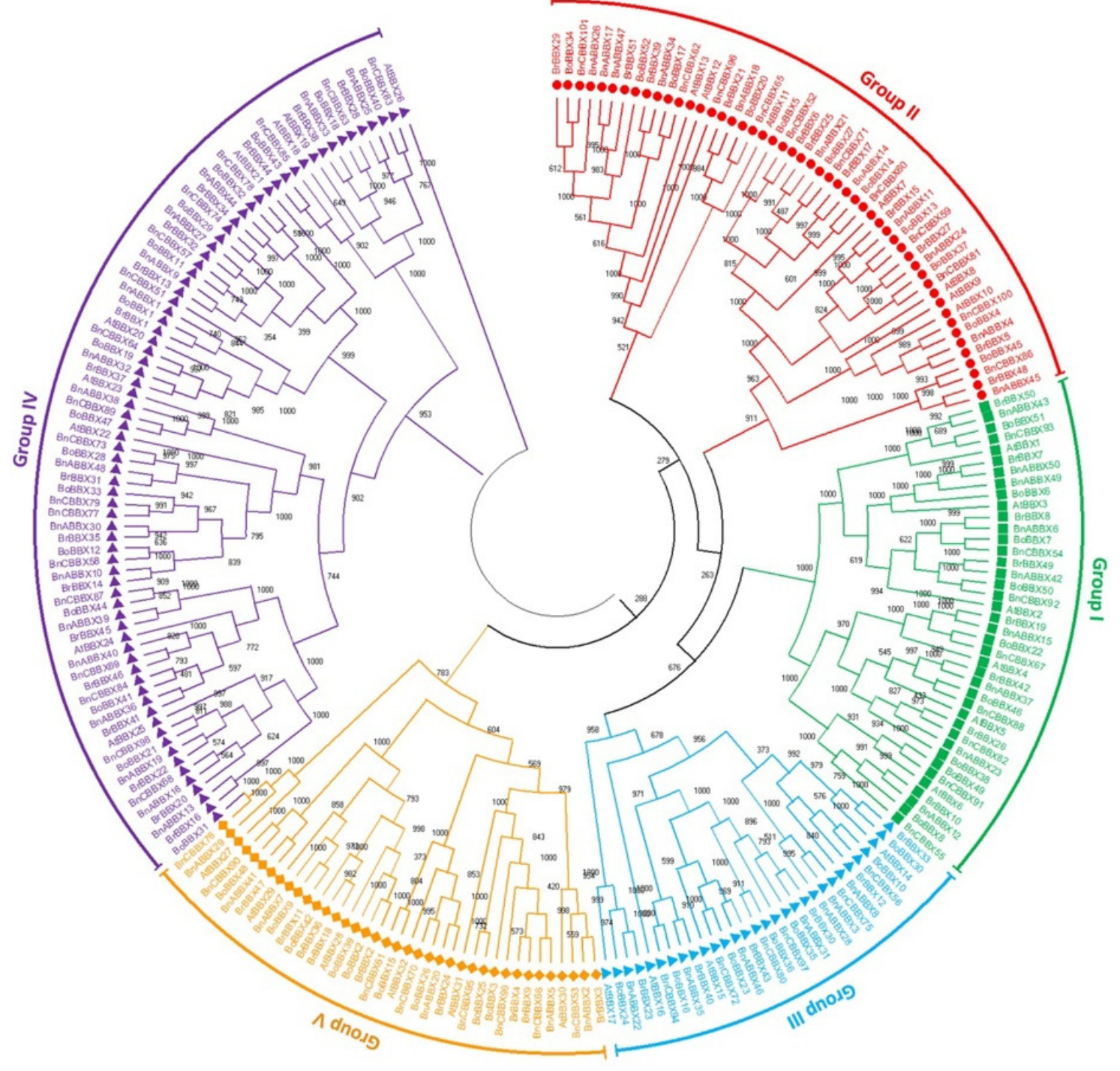

Figure 2. Phylogenetic tree of B-box genes from Brassica rapa, B. napus, B. oleracea, and Arabidopsis thaliana. The phylogenetic tree was constructed according to the neighbor-joining method. The unrooted tree was generated using MEGA5 on the basis of B-box amino acid sequences from B. rapa, B. napus, B. oleracea, and A. thaliana. Roman numerals (I-V) represent different gene clusters. The genes from each species are differentiated by color. The numbers at the nodes represent bootstrap percentage values.

The protein sequence alignment is shown in Supplementary Figure S1a-c. The protein logos were generated for all Brassica BBXs depicted in Figure 3. This analysis confirms the structural integrity of the domains and indicates that the B1 (Figure 3a), B2 (Figure 3b), and CCT (Figure 3c) domains were well conserved among B. rapa, B. oleracea, and B. napus BBX proteins. 
a

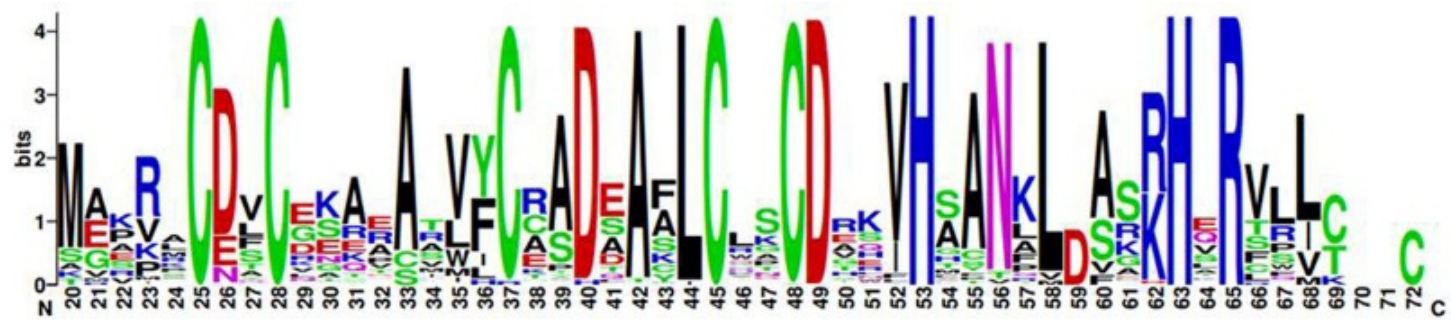

b

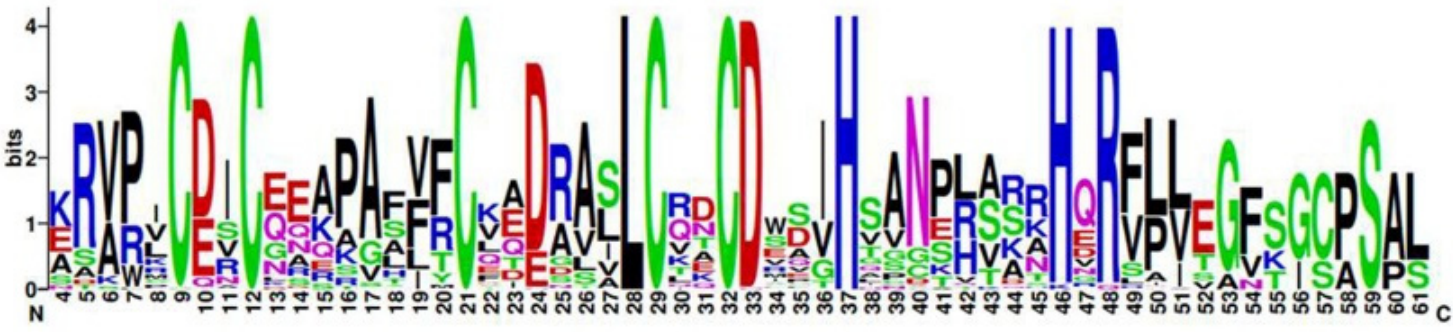

c

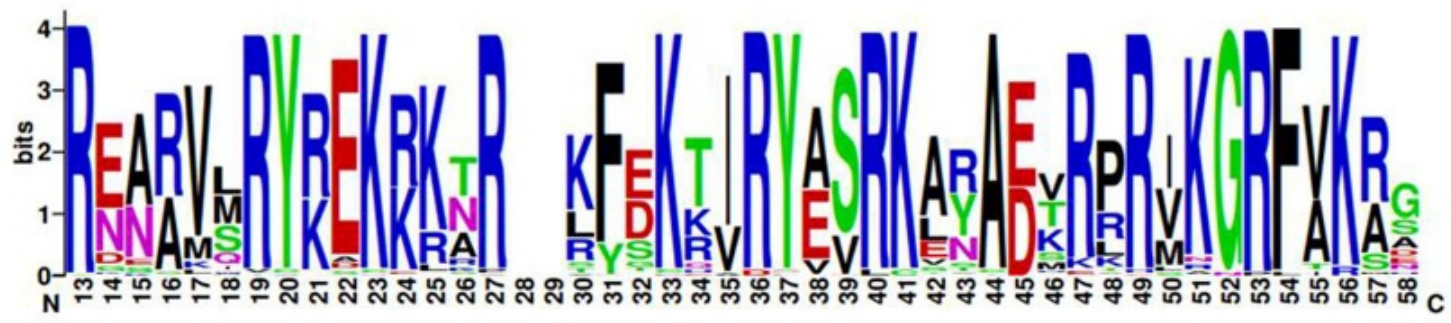

Figure 3. Web logos of BrBBX proteins. (a-c) Alignments of the B-box1, B-box2, and CCT domains, respectively. The $x$-axis indicates the conserved domain sequences. The height of each letter indicates how conserved the residue is across all proteins. The $y$-axis presents the relative entropy, which reflects the conservation rate of each amino acid.

For each examined Brassica species, the phylogenetic tree was divided into five classes (Classes I-V). In B. rapa, Class IV (B1 + B2) was the largest (17 BBXs). In contrast, Classes II and $\mathrm{V}$ comprised eleven and nine BBXs each, whereas Classes I and III included eight and nine BBXs each (Figure 4a). Similarly, in B. oleracea, Class IV was the largest (16 BBXs) and Class I and V contained nine BBXs, while Class II and III comprised eleven and seven BBXs (Figure 5a). Regarding B. napus, Class IV included $36 \mathrm{BBX}$, which was more than the 26, 14, 13, and 12 BBXs in Classes I, V, II, and III, respectively (Figure 6a). Accordingly, Class IV (B1 + B2) was the largest for all three analyzed Brassica species. Class III (B1 + CCT) was the smallest class. 

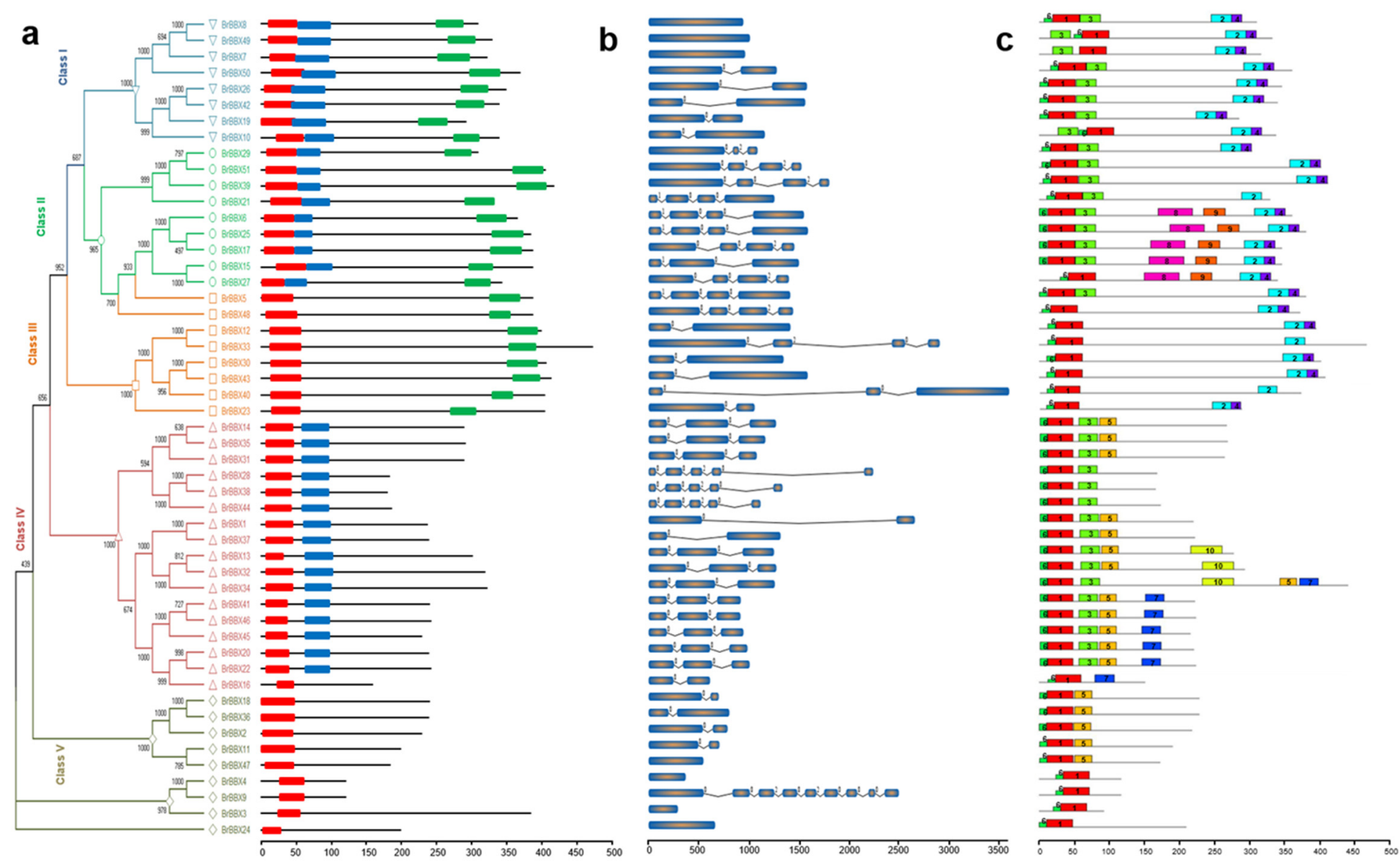

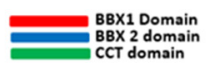

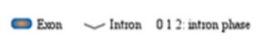

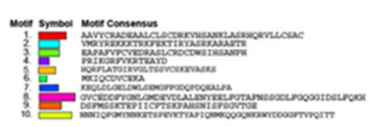

Figure 4. Brassica rapa B-box gene structures (a), motifs (b), and conserved domains (c). The domain architecture of the B-box genes is presented in panel a. Exons are represented by colored boxes, whereas introns are indicated by gray lines $(\mathbf{b})$. Colored boxes indicate conserved motifs and gray lines represent non-conserved sequences. The motif lengths in each protein are presented proportionally. Classes I-V represent different groups of B-box family members in B. rapa. 
a

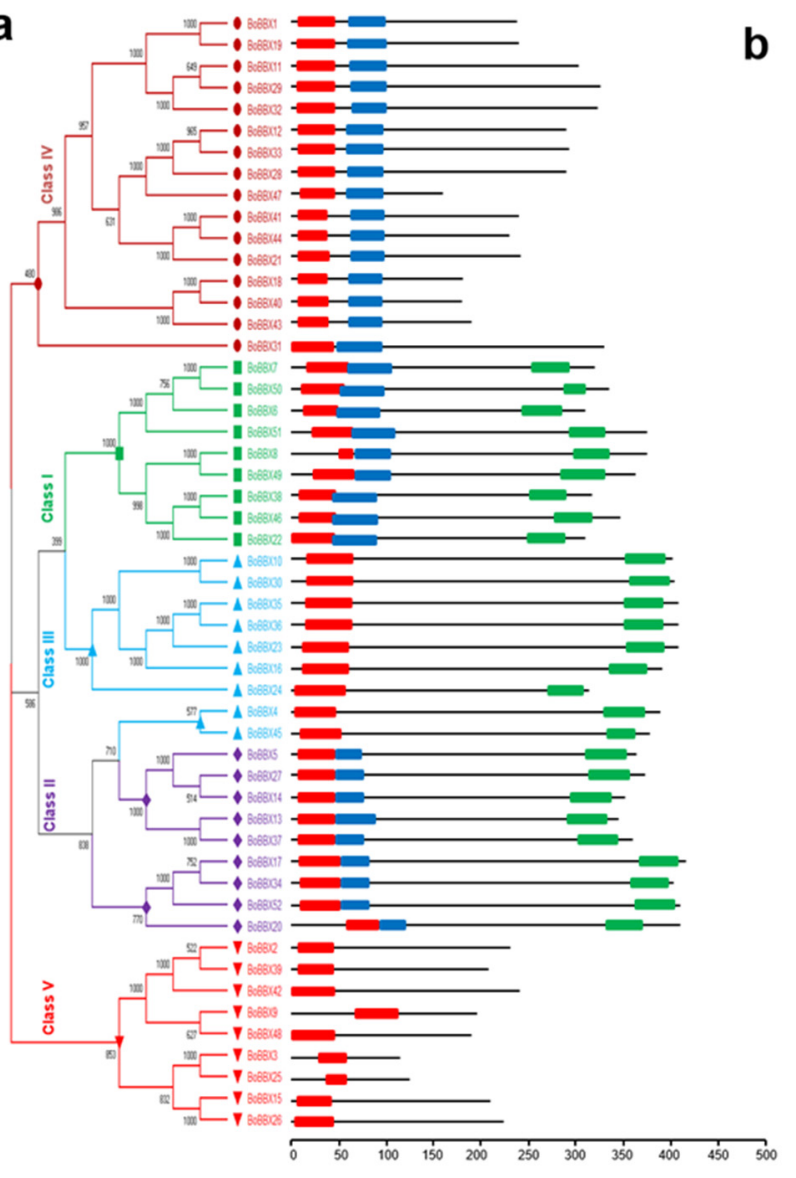

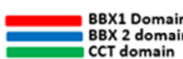

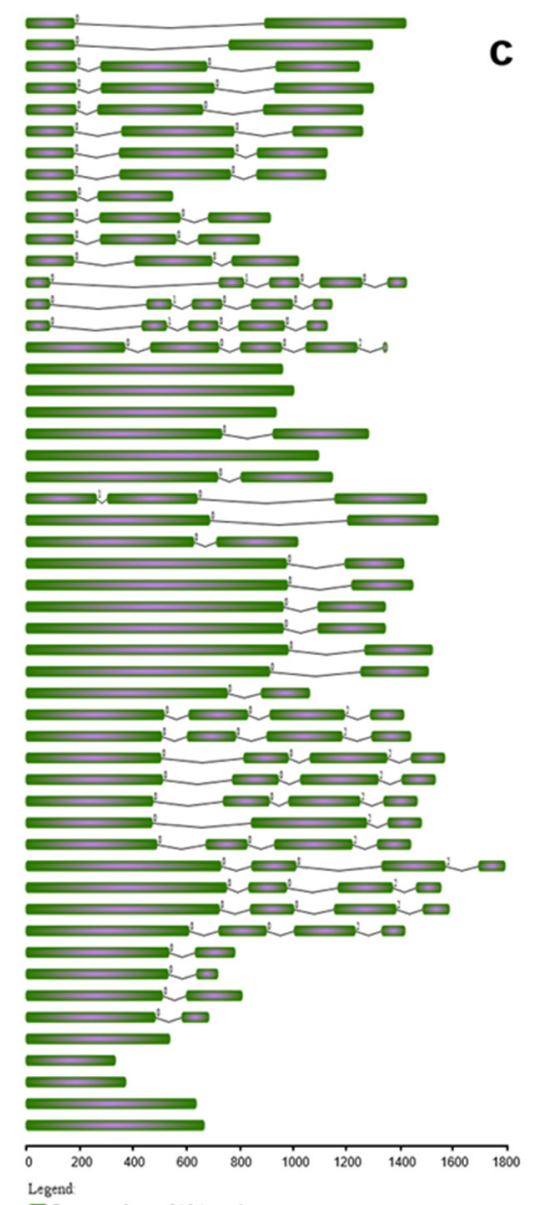

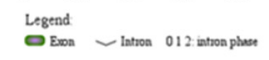

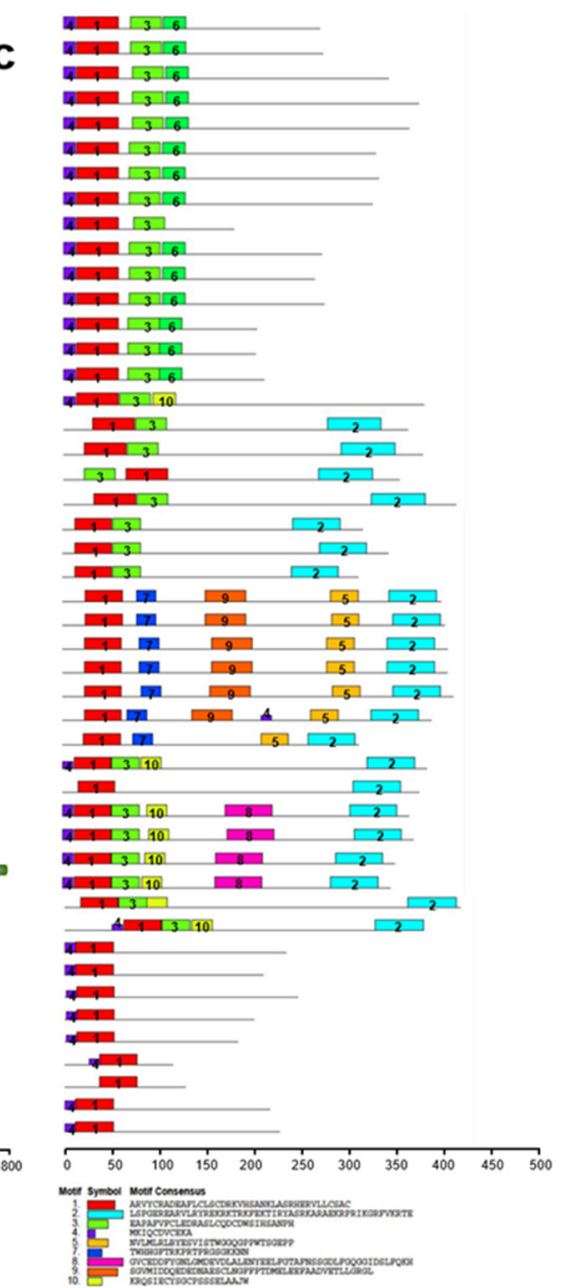

Figure 5. Brassica oleracea B-box gene structures (a), motifs (b), and conserved domains (c). The domain architecture of the B-box genes is presented in panel a. Exons are represented by colored boxes, whereas introns are indicated by gray lines $(\mathbf{b})$. Colored boxes indicate conserved motifs and gray lines represent non-conserved sequences. The motif lengths in each protein are presented proportionally. Classes I-V represent different groups of B-box family members in B. oleracea. 


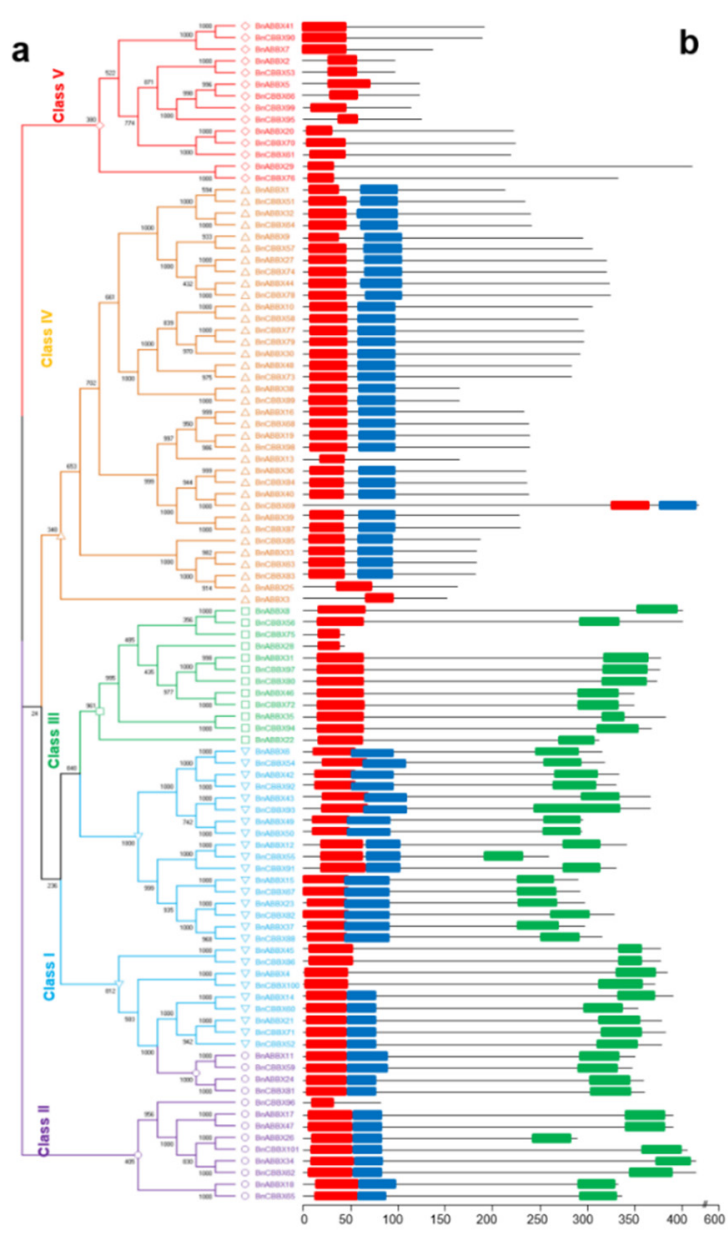

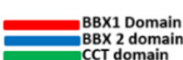

b

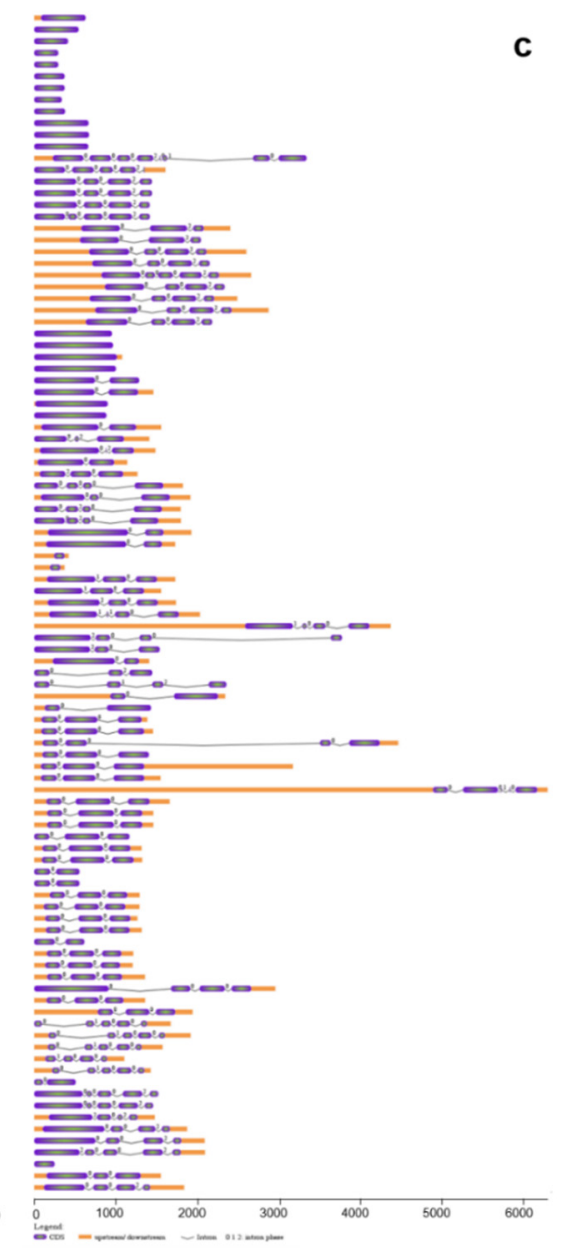

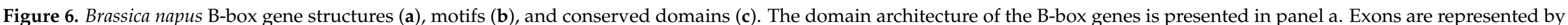

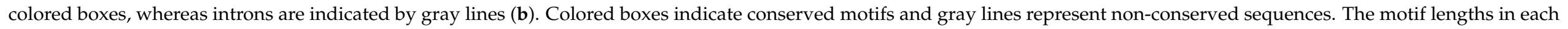
protein are presented proportionally. Classes I-V represent different groups of B-box family members in B. napus. 
Different combinations of introns and exons lead to diverse gene functions. The structural diversity among the BBX genes in the three Brassica species was analyzed to clarify the evolution of the Brassica BBX gene families (Figures $4 b$ and $5 b$ ). The B. rapa BBX genes had 1-9 exons (Figure 4b). Most Class I genes had one or two exons, whereas the Class II genes contained three or four exons. Most of the Class III genes comprised two or four exons; the exception was BrBBX40 (three exons). In Class IV, the majority of the genes contained three or five exons; the exceptions were $B r B B X 1, B r B B X 37$, and $B r B B X 16$ (two exons). In Class $\mathrm{V}$, most genes harbored one or two exons, but $B r B B X 9$ had nine exons (Figure $4 b$ ). The B. oleracea BBX genes included 1-5 exons (Figure $5 b$ ). Similar to the Class I $B$. rapa genes, most of the Class I $B$. oleracea genes contained one or two exons; the exception was BoBBX38 (three exons). In Class II, all genes harbored four exons, except for BoBBX13 (three exons). Most of the Class III genes contained two or four exons, whereas the Class IV genes comprised 2-5 exons. In Class V, almost all genes had one or two exons, similar to the Class V B. rapa genes (Figure 5b). In B. napus, the BBX genes comprised 1-5 exons (Figure $6 \mathrm{~b}$ ). In Class I, the majority of genes contained 2-5 exons, which differed from the Class I genes in B. rapa and B. oleracea (one or two exons). Most of the Class II genes harbored 3-5 exons; the exception was BnCBBX62 (one exon). In Class III, most genes contained 2-4 exons. The Class IV genes contained 1-5 exons. The Class V genes harbored a single exon, with the exception of $B n A B B X 29$ (seven exons) and BnCBBX76 (four exons) (Figure 6b). These findings reflect the structural similarity among the Brassica BBX genes as well as the gain and loss of exons during evolution.

We investigated the motif distribution in the BBX genes from the three examined Brassica species to functionally characterize the genes (Figures $4 c, 5 c$ and $6 c$ ). In B. rapa, 10 motifs were detected, of which motifs 1 and 6 were conserved (except in BrBBX7) (Figure 4c). In Classes I and II, almost all genes encoded motifs 1-4 and motif 6 . Five of the Class II genes also included motifs 8 and 9. The Class III genes included motifs 1 , 2, 4 and 6, but lacked motif 3 (except for BrBBX29). In Class IV, which was the largest (17 $B r B B X$ genes), most of the genes contained motifs 1, 3, 5 and 6 , whereas some of the genes also included motif 7 (seven $B r B B X$ genes) and motif 10 (three $B r B B X$ genes) (Figure 4c). In B. oleracea, among the 10 identified motifs, motif 1 was conserved in all 52 BBX genes (Figure 5c). Motifs 1 and 2 were common among the genes in Classes I, II and III. In contrast, motifs 6 and 10 were, respectively, detected in only BoBBX7 and BoBBX50 in Class I. Motifs 8 and 10 (except in BoBBX50) were exclusive to the Class II genes. In Class III, most of the genes contained motifs 5, 7, and 9. In Class IV, the genes harbored motifs 1 , 3,4 , and 6 (with the exception of BoBBX41). The Class V BBX genes encoded motifs 1 and 4 (with the exception of BoBBX25) (Figure 5c). The B. napus BBX genes included 10 motifs, of which motif 5 was conserved in all 101 genes (Figure 6c). In Class I, motifs 3, 5, and 9 were conserved in all genes, and motif 10 was detected in some of the genes. Class II contained all 10 motifs, with motifs 4 and 5 in most genes and motif 7 included in five genes. In Class III, motif 1 (except in $B n C B B X 81$ ), motif 2 (except in five BnBBX genes), and motif 4 (except in five $B n B B X$ genes) were conserved, whereas motif 7 was included in only four $B n B B X$ genes. The 36 genes in Class IV were missing motifs 9 and 10, but motif 7 was included in nine $B n B B X$ genes. In Class $V$, along with motif 5 , we detected motif 3 in five $B n B B X$ genes. Moreover, motif 1 was not detected in $B n A B B X 20, B n C B B X 70$, and $B n C B B X 61$ (Figure 6c).

\subsection{Chromosomal Distribution of the B. rapa, B. oleracea, and B. napus BBX Genes}

We observed that 50 of $51 \mathrm{BrBBX}$ genes, 51 of $52 \mathrm{BoBBX}$ genes, and 79 of $101 \mathrm{BnBBX}$ genes were randomly distributed among the chromosomes in B. rapa (Figure S2a), B. oleracea (Figure S3a), and B. napus (Figure S4a), respectively. One B. rapa gene (BrBBX51), one $B$. oleracea gene (BoBBX52), and 22 B. napus genes were not mapped on any chromosome. In B. rapa, chromosome A02 harbored the most BrBBX genes (eight), followed by chromosomes A01 and A07, both of which had seven BrBBX genes (Figure S2a). Chromosome A04 contained the fewest $B r B B X$ genes (two). The BrBBX genes were mapped on all 10 chromosomes, reflecting their broad chromosomal distribution. In B. oleracea, 
chromosomes $\mathrm{C} 02$ and $\mathrm{C} 07$ had the most BoBBX genes (seven), followed by chromosomes C01, C03, C06 and C09, which contained six BoBBX genes (Figure S3a). Similar to B. rapa, chromosome C04 in B. oleracea had the fewest BoBBX genes (three). The B. napus BnBBX genes were mapped on the AA and $C C$ genomes. In the A genome, chromosome A02 contained the most $B n B B X$ genes (seven) (similar to $B$. rapa and B. oleracea), followed by chromosomes A07 (six) and A08 (five) (Figure S4a). Similar to B. rapa and B. oleracea, chromosome A04 had the fewest $B n B B X$ genes (three). Three or four genes were clustered at the end of chromosomes A04, A06, and A08. In the C genome, chromosome C06 contained the most $B n C B B X$ genes (seven), followed by chromosomes $C 02$ and $C 09$, which had six $B n B B X$ genes (Figure S4a). Unlike $B$. rapa and $B$. oleracea, the fewest $B n B B X$ genes (three) were mapped on chromosome C01 (Figure S4a). The distribution analysis indicated the BBX genes were most abundant on chromosome 2 in all examined Brassica genomes, possibly because of gene duplication events.

\subsection{Analyses of Synteny and Duplication of BBX Orthologous Genes}

Whole-genome and tandem duplications are critical events for enhancing genome complexity and the evolution of novel gene functions. Only one gene pair (single tandem array) was associated with a tandem duplication in the B. rapa, B. oleracea, and B. napus genomes (Table S4). Single tandem duplication events were detected in B. rapa (chromosome A10) involving Bra008668 and Bra008669 as well as in B. oleracea (chromosome C09) involving Bo9g163720.1 and Bo9g163730.1. In B. napus, BnaC09g41980D and BnaC09g41990D on chromosome C09 were the result of a single duplication event (Table S4).

Thirty gene pairs in B. rapa (Figure S2b; Table S5), 27 gene pairs in B. oleracea (Figure S3b; Table S6), and 64 gene pairs in B. napus (Figure S4b; Table S7) were segmentally duplicated. Our analysis also revealed the variability in the number of duplicated genes per segmental duplication event and an uneven distribution of these duplications on seven of 10 chromosomes in B. rapa, six of nine chromosomes in B. oleracea, and 17 of 19 chromosomes in $B$. napus (Tables S5-S7). In B. rapa, chromosomes A01 and A02 had the most gene pairs (nine and eight, respectively), whereas chromosomes A06 and A09 contained the fewest gene pairs (one each). In B. oleracea, chromosomes C02 and C08, respectively, had the most (nine) and fewest (one) gene pairs. In B. napus, chromosomes A02 and A08 contained the most gene pairs (10 and 11, respectively), whereas chromosomes A01, C01, C03, C05, C07, and C08 had the fewest gene pairs (one each). Additionally, the B. napus A genome included more segmentally duplicated gene pairs than the C genome (Tables S5-S7).

\subsection{Comparative Synteny Analysis of Orthologous Pairs of BBX Genes in Brassicaceae}

In addition to identifying gene duplication events, we investigated the synteny between the BBX gene families of $A$. thaliana, B. rapa, B. oleracea, and B. napus. A total of 43 chromosomes $(5,10,9$, and 19 from $A$. thaliana, B. rapa, B. oleracea, and B. napus, respectively) and $236 \mathrm{BBX}$ genes $(32,51,52$, and 101 from $A$. thaliana, B. rapa, B. oleracea, and $B$. napus, respectively) were used to investigate syntenic relationships. Orthologous BBX genes and genes resulting from segmental duplications are indicated by different colored lines in Figure 7. The BrBBX genes on chromosomes 1 (BrA01), 2 (BrA02), and 10 (BrA10) had orthologs on chromosomes AT1, AT3, AT4, and AT5 (A. thaliana), C01, C02, and C09 (B. oleracea), and A01, A02, A08, A09, A10, C01, C02, C06, C08 and C09 (B. napus). A total of 235 BBX gene pairs (Tables S8-S13) had syntenic relationships between $A$. thaliana and $B$. rapa, B. oleracea, and $B$. napus. Additionally, 58 orthologous pairs were detected between $A$. thaliana and $B$. rapa as well as between $A$. thaliana and B. oleracea, which was fewer than the 68 orthologous pairs detected between $A$. thaliana and B. napus. Fifty-six orthologous pairs were identified between $B$. rapa and $B$. oleracea as well as 121 orthologous pairs between $B$. rapa and $B$. napus and 123 orthologous pairs between $B$. oleracea and $B$. napus (Tables S8-S13). 


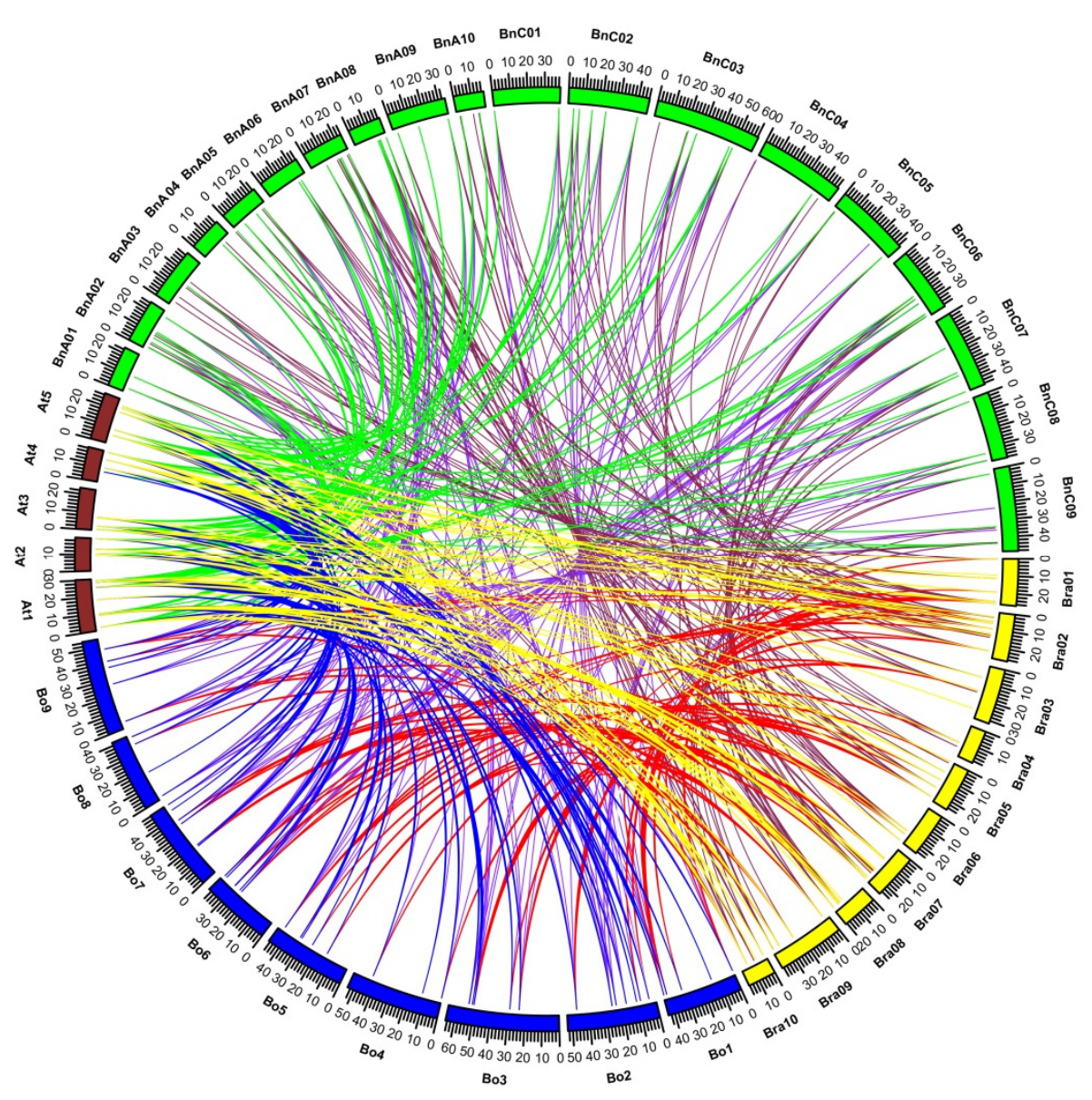

Figure 7. Brassica rapa (Bra01-Bra10), B. oleracea (C01-C09), B. napus (Bna01-Bna10 and Bnc01-Bnc09), and Arabidopsis thaliana (At1-At5) chromosomal maps were constructed according to orthologous gene pair positions. The maps revealed highly conserved synteny. The orange, blue, and red curves link the BBX genes on $A$. thaliana chromosomes 1, 2, 3, 4, and 5 with their orthologous genes in B. rapa, $B$. oleracea, and $B$. napus, respectively.

Of the 58 orthologous gene pairs between A. thaliana and B. rapa, eight AtBBX genes had a single copy of an orthologous gene in $B$. rapa. In contrast, there were 2-4 copies of the other AtBBX orthologs in B. rapa (Table S8). More specifically, there were two copies of seven $A t B B X$ orthologs, three copies of nine AtBBX orthologs, four copies of the AtBBX24 ortholog, and five copies of the $A t B B X 28$ ortholog in B. rapa. An analysis of the synteny among orthologs revealed the genes generally belonged to the same groups. For example, AtBBX1 (Group I) was syntenic with the Group I genes $B r B B X 7, B r B B X 8$, and $B r B B X 49$. However, there were some exceptions. Specifically, $A t B B X 9$ and $A t B B X 10$, which belong to Group II (B1 + B2 + CCT), had syntenic relationships with BrBBX5, which belongs to Group III $(\mathrm{B} 1+\mathrm{CCT})$, indicative of a loss of the B2 domain during evolution. Altogether, 26 A. thaliana BBX genes were syntenic with 46 B. rapa genes (Table S8).

An examination of the 58 orthologous gene pairs between $A$. thaliana and B. oleracea indicated that the $B$. oleracea genome included single copies of eight $A t B B X$ orthologs (Table S9). Interestingly, there were single copies of the AtBBX4, AtBBX9, AtBBX10, $A t B B X 11$, and $A t B B X 17$ orthologs in both $B$. rapa and B. oleracea, reflecting their identical genomic arrangements. Among the other orthologous gene pairs, there were two copies of $12 A t B B X$ orthologs (segmental duplications), three copies of six AtBBX orthologs, and four copies of AtBBX15 and AtBBX28 orthologs in B. oleracea (Table S9). Moreover, the orthologous gene pairs belonged to the same groups (according to their domains), with the exception of the $A t B B X 9$ and $A t B B X 10$ orthologs in B. oleracea, which lacked the B2 domain. A comparative analysis of $A$. thaliana and $B$. napus indicated that of the 68 orthologous gene 
pairs, there was a single copy of four $A t B B X$ orthologs, two and four copies of six $A t B B X$ orthologs, and three copies of three $A t B B X$ orthologs in B. napus (Table S10). Although $A t B B X 9$ and $A t B B X 10$ orthologs were detected in B. rapa and B. oleracea, they were missing in B. napus.

We detected 56 orthologous gene pairs between B. rapa and B. oleracea. A single copy of nine $B r B B X$ orthologs, two copies of $13 B r B B X$ orthologs, and three copies of seven $B r B B X$ orthologs were detected in B. oleracea (Table S11). A total of 29 B. rapa genes had syntenic relationships with $28 \mathrm{~B}$. oleracea genes. The $B r B B X 6$ gene, which belongs to Group II $(\mathrm{B} 1+\mathrm{B} 2+\mathrm{CCT})$, was syntenic with $B n A B B X 14, B n A B B X 21, B n C B B X 52, B n C B B X 60$, and $B n C B B X 71$, which belong to Group I (Tables S11-S13). Of the 121 orthologous gene pairs between $B$. rapa and $B$. napus, there was a single copy of seven $B r B B X$ orthologs, two copies of $11 B r B B X$ orthologs, as well as three, four, and five copies of 9, 10, and 5 BrBBX orthologs, respectively, in B. napus (Table S12). Moreover, BrBBX9 was syntenic with the exostosin genes in B. napus (BnaA03g06300D, BnaA10g17600D, BnaC03g08100D, and BnaC09g40970D), whereas BrBBX10, BrBBX24, BrBBX33, and BrBBX48 (Table S12) were syntenic with the B. napus CCT superfamily genes (BnaA10g11600D, BnaA03g36070D, BnaA07g24620D, BnaA10g03390D, and BnaC05g03370D), which included only the CCT domain (Table S12). Overall, 42 B. rapa BBX genes were syntenic with 71 B. napus genes.

A comparative analysis of the common orthologous pairs of BBX genes among four species (B. rapa, A. thaliana, B. oleracea, and B. napus) identified orthologs of 26 B. rapa BBX genes in the A. thaliana, B. oleracea, and B. napus genomes (Figure 8; Table S14). Almost all of the BBX genes had a one-to-one relationship between the B. rapa and A. thaliana genomes; the exceptions were the Bra008668, Bra010994, Bra023541, and Bra031180 orthologs in A. thaliana (two copies each). However, the comparison with B. oleracea indicated there were two or three copies of most BBX genes. Only six BBX genes were detected as single copies. Furthermore, there were multiple copies (between two and five) of almost all B. rapa and $A$. thaliana BBX orthologs in B. napus. The exception was the Bra004035 ortholog (single copy).

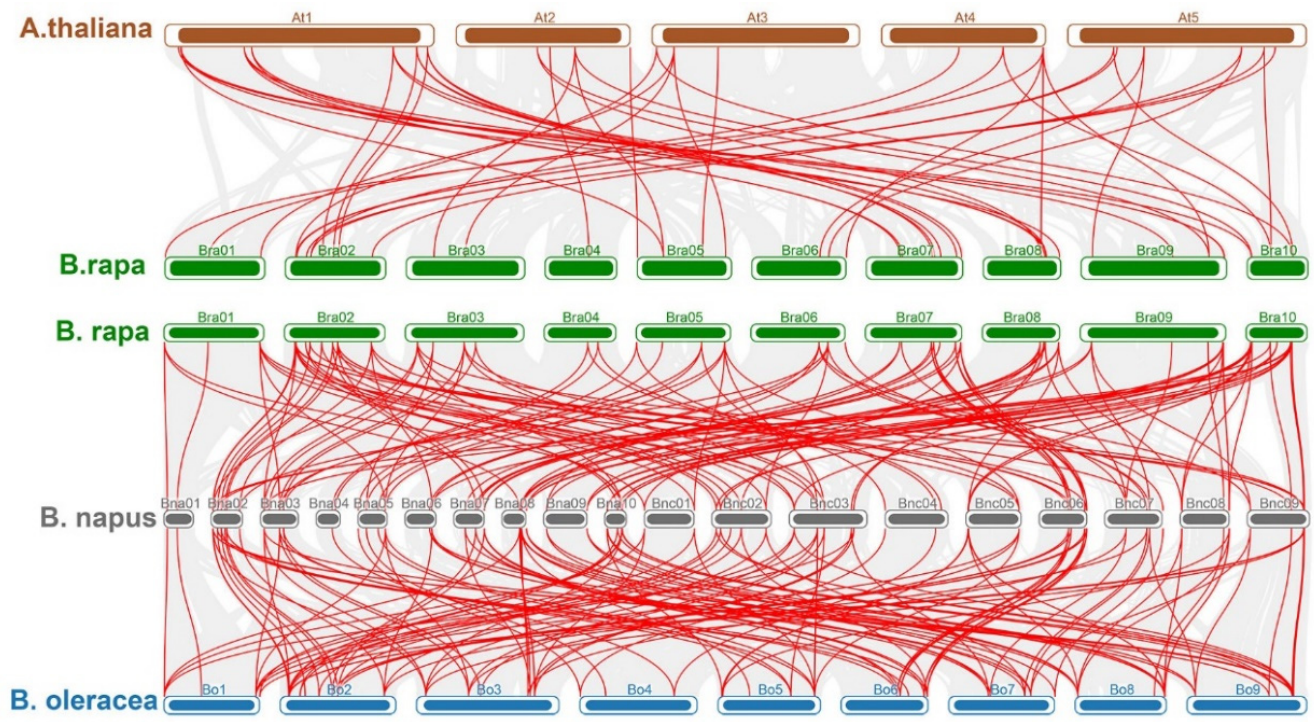

Figure 8. Syntenic collinearity relationship of B-box genes among A. thaliana and B. rapa, B. oleracea, and $B$. napus. In the background, gray lines designate the collinear blocks within the $A$. thaliana and other Brassica genomes. Diverse colored lines indicate the relationships among orthologous gene pairs between four Brassica species. 
2.6. Analysis of the Ka and Ks Values of the Orthologous BBX Genes among Brassica Species and Arabidopsis Thaliana

The non-synonymous mutation rate $(\mathrm{Ka})$ and the synonymous mutation rate $(\mathrm{Ks})$ were calculated to determine the selection pressure associated with the duplication of BBX genes in Brassica species (Tables S8-S10). First, the orthologous BBX genes among A. thaliana and the three Brassica species were identified. The 58, 56, and 121 pairs of orthologous BBX genes between $A$. thaliana and B. rapa, B. oleracea, and B. napus, respectively, were used to calculate the $\mathrm{Ka}, \mathrm{Ks}$, and $\mathrm{Ka} / \mathrm{Ks}$ values (Tables S8-S10). Most of the $\mathrm{Ka} / \mathrm{Ks}$ values for the Brassica species were less than 1, indicating the orthologous genes were under strong purifying selection pressure. The $\mathrm{Ka} / \mathrm{Ks}$ value exceeded 1 for one orthologous gene pair between B. rapa and B. napus (Bra003748 and BnaA07g21640D), reflecting positive selection pressure.

\subsection{Cis-Elements in the Brassica BBX Gene Promoters}

Cis-acting elements in the promoter region are crucial for regulating the expression of the corresponding gene because they bind with various transcription factors [50]. Thus, we identified the cis-regulatory elements in 1500-bp sequences upstream of the start codon of genes in B. rapa, B. oleracea, and B. napus using the PlantCARE database (Table S15). As predicted, common promoter elements (e.g., TATA-box and CAAT-box) were identified in all Brassica BBX gene promoters. Several cis-elements related to plant development and growth, phytohormone responses, light responses, and stress responses were detected in all three Brassica species. The following 15 light-responsive cis-elements involved in growth and development were detected in the BBX gene promoter regions: ARE, G-box, ABRE, Box4, GT1-motif, AE box, MRE, ACE, I-box, chs-CMA, GA motif, GAP-box, 3-AF1 binding site, Sp1, and MSA-like (Table S15). Among the cis-acting elements involved in hormone responses, ABRE, ERE, GAREs (GARE-motif and P-box), and the MeJAresponsive elements (CGTCA-motif and TGACG-motif) were, respectively, identified in the promoter regions of 41, 31, 27, and 62 BBX genes in B. rapa, 42, 28, 32, and 81 BBX genes in B. oleracea, and 86,55,64, and $143 \mathrm{BBX}$ genes in $B$. napus. The zein metabolism regulation element (O2 site) was detected in $12 \mathrm{BrBBX}, 21 \mathrm{BoBBX}$, and $31 \mathrm{BnBBX}$ genes, whereas the CAT-box influencing meristem expression was identified in the promoter regions of $18 B r B B X, 22 B o B B X$ and $39 B n B B X$ genes.

Of the stress-related response elements, ARE, which is an anaerobic induction element, was detected in the promoters of most BBX genes. Some stress-related (low temperatures, wounding, and drought) cis-acting elements were also identified in the promoter regions of BBX genes (Table S15).

\subsection{Developmental and Tissue-Specific Expression of BrBBX Genes}

We investigated the expression patterns of $51 \mathrm{Br} B B X$ genes in different organs and during various developmental stages using available transcriptome data [51]. Gene expression was analyzed in the callus, flower, leaf, root, silique, and stem. The expression levels varied among genes, with some genes exhibiting tissue-specific expression (Figure 9). These results suggest that BBXs in $B$. rapa may affect diverse biological processes in different tissues. Of the $51 B r B B X$ genes, 12 (BrBBX19, BrBBX21, BrBBX25, BrBBX29, BrBBX6, BrBBX35, $B r B B X 39, B r B B X 41, B r B B X 44, B r B B X 45, B r B B X 46$ and $B r B B X 51)$ were relatively highly expressed in all developmental stages and tissues, reflecting their overall involvement in $B$. rapa plant development. Some BBX genes (BrBBX5, BrBBX16, BrBBX40, BrBBX48 and $B r B B X 51)$ were expressed at very low levels in all organs in different growth stages. Additionally, $B r B B X 27, B r B B X 32$, and $B r B B X 37$ were expressed at higher levels in the silique than in the stem, flower, leaf, and root (Figure 9). 


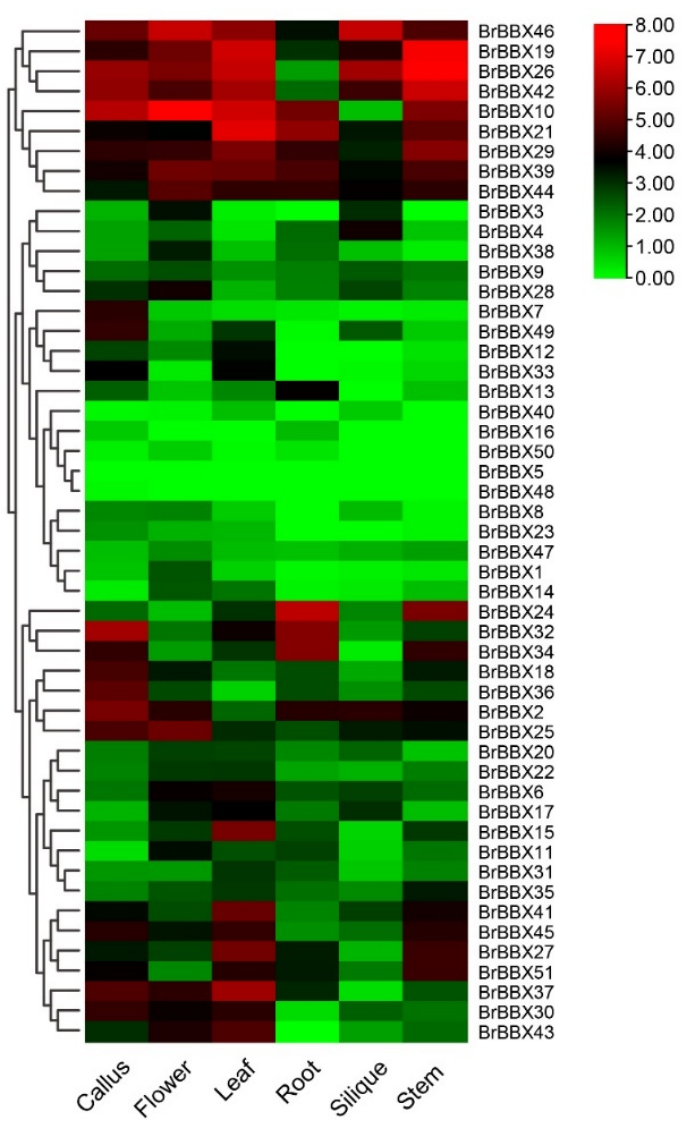

Figure 9. Tissue-specific $B r B B X$ expression patterns. The relative expression data were $\log _{2}$ transformed using the pheatmap package of R. A cluster dendrogram is provided to the left of the heat map. Blue and red indicate downregulated and upregulated expression, respectively.

Similarly, $B r B B X 26, B r B B X 30$, and $B r B B X 43$ were expressed exclusively in the root, whereas $B r B B X 7$ was expressed only in the callus. The $B r B B X 10$ gene was highly expressed in all organs, except for the silique, and $B r B B X 19, B r B B X 21$, and $B r B B X 26$ were expressed at high levels in all organs. The other BBX genes were expressed at lower levels in the various organs. Moreover, the genes in the same group had identical expression patterns.

\subsection{BrBBX Expression Patterns in Response to Various Abiotic Stresses and Hormones}

Abiotic stresses and hormones substantially influence plant development and growth. To clarify the effects of different abiotic stresses and hormones on the expression of Brassica $B B X$ genes, the BBX gene expression patterns at various time points were analyzed by qRT-PCR. We randomly selected $12 \mathrm{Br} B B X$ genes $(B r B B X 4, B r B B X 6, B r B B X 10, B r B B X 13$, BrBBX14, BrBBX15, BrBBX17, BrBBX22, BrBBX24, BrBBX26, BrBBX33 and BrBBX48) from five groups and examined their expression following an exposure to different abiotic stresses and hormones (i.e., $\mathrm{NaCl}$, mannitol, PEG, cold, heat, drought, ABA, SA and MeJA). The BrBBX15 and BrBBX17 expression levels were upregulated by all abiotic stresses and exogenous hormone treatments (i.e., compared with the samples at $0 \mathrm{~h}$ ) (Figures 10-12). The most upregulated gene was $B r B B X 6$ at $24 \mathrm{~h}$ after the mannitol treatment (104-fold increase). Additionally, $B r B B X 6$ was also highly expressed in response to $\mathrm{NaCl}(6 \mathrm{~h})$ and $\mathrm{PEG}(12 \mathrm{~h})$ (65-fold and 21-fold increases, respectively). The qRT-PCR analysis indicated that $B r B B X$ expression levels were affected by $\mathrm{NaCl}$, mannitol, and cold stress more than by PEG, heat, and drought (Figures 10 and 11). Exposure to heat stress generally downregulated the expression of $B r B B X$ genes, with the exception of $B r B B X 13, B r B B X 15, B r B B X 17$ and BrBBX33. 


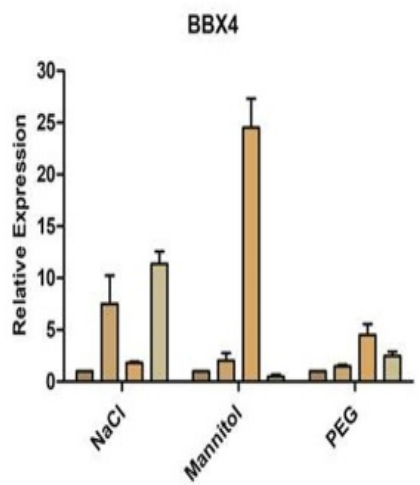

BBX14

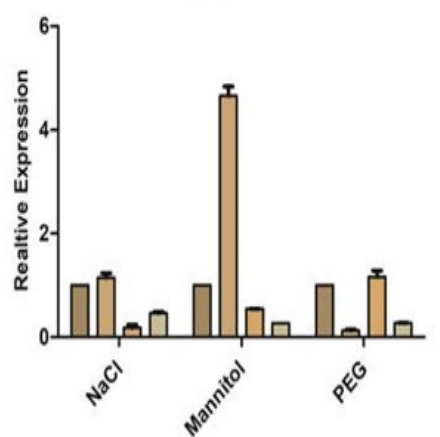

BBX24

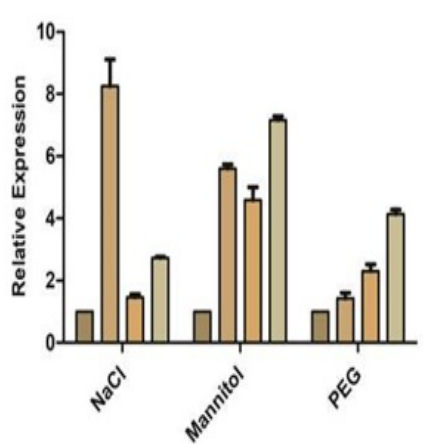

BBX6

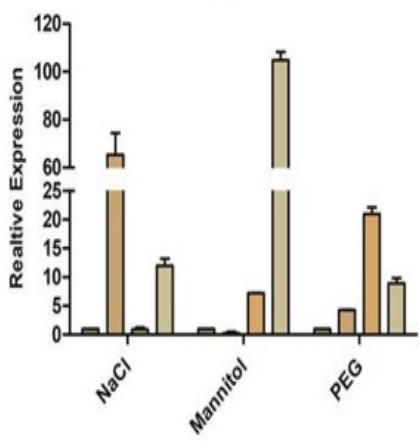

BBX15

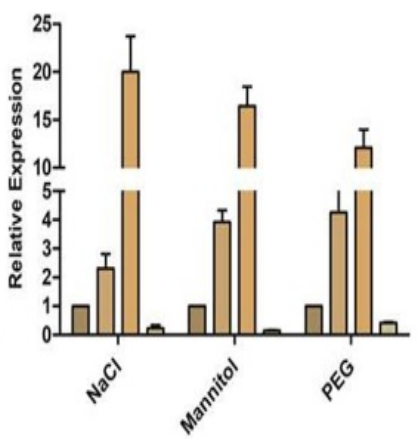

BBX26

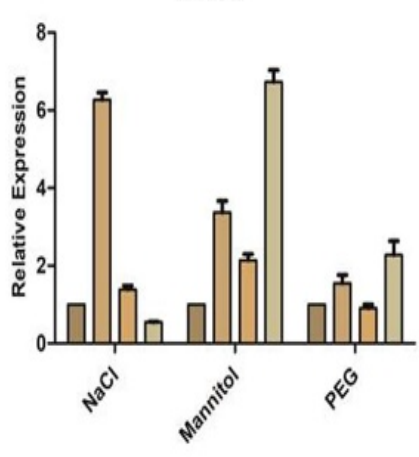

BBX10

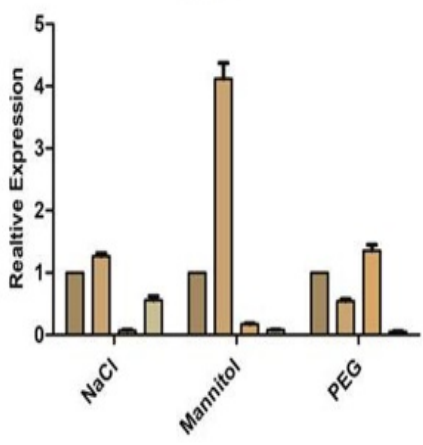

BBX17
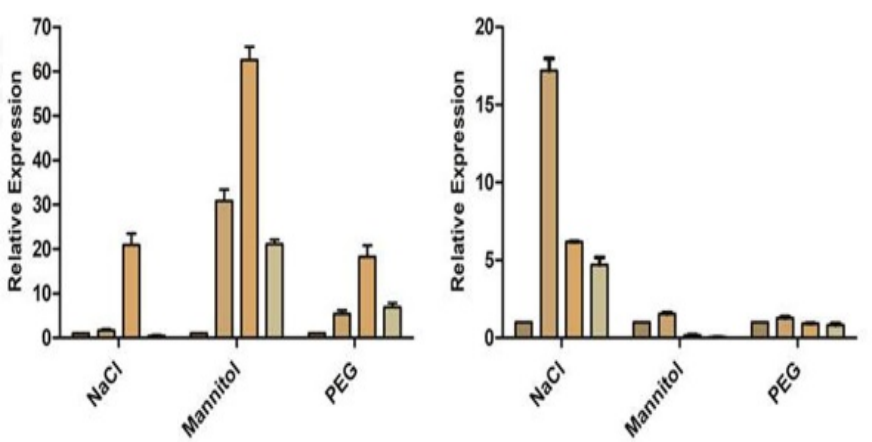

BBX33

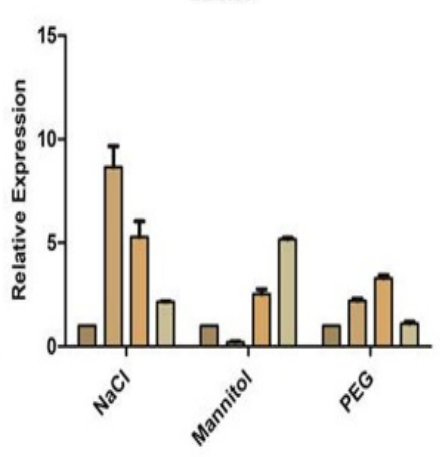

BBX13

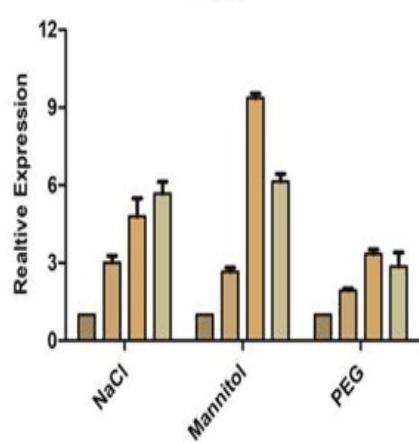

BBX22

Figure 10. Abiotic stress-induced BrBBX expression profiles. The graphs present the expression levels in Brassica plants following $\mathrm{NaCl}$, mannitol, and PEG treatments. Samples were collected at various time points for a qRT-PCR analysis. The $x$-axis presents the abiotic stresses. The $y$-axis presents the relative expression levels. Error bars indicate the standard deviations of three independent qRT-PCR biological replicates. 
BBX4

BBX 6

BBX10

BBX13
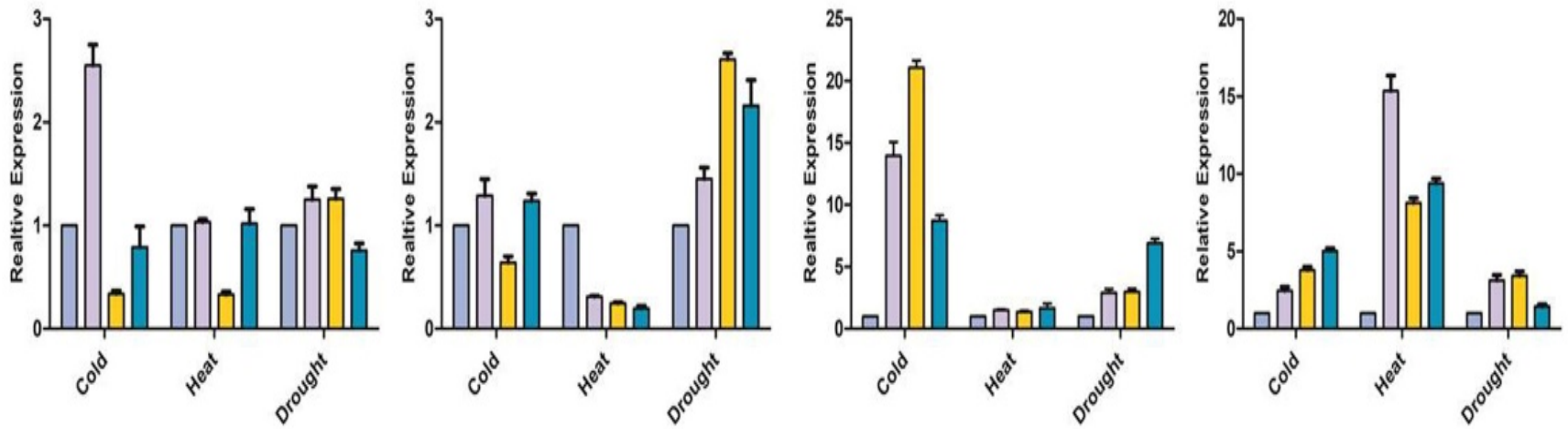

BBX14

BBX15

BBX17

BBX22
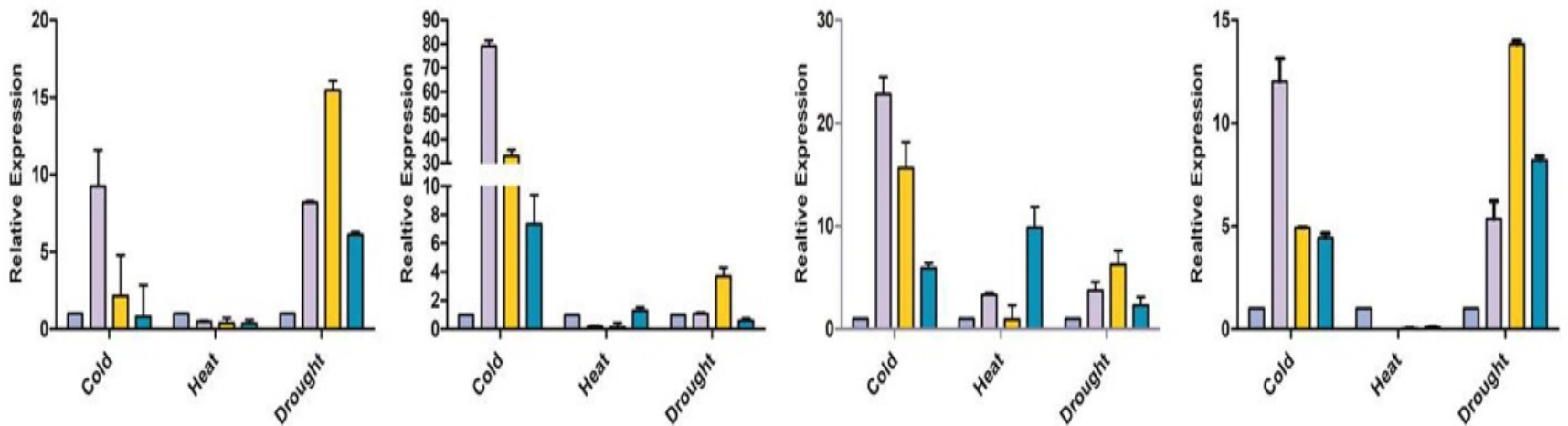

BBX24

BBX26
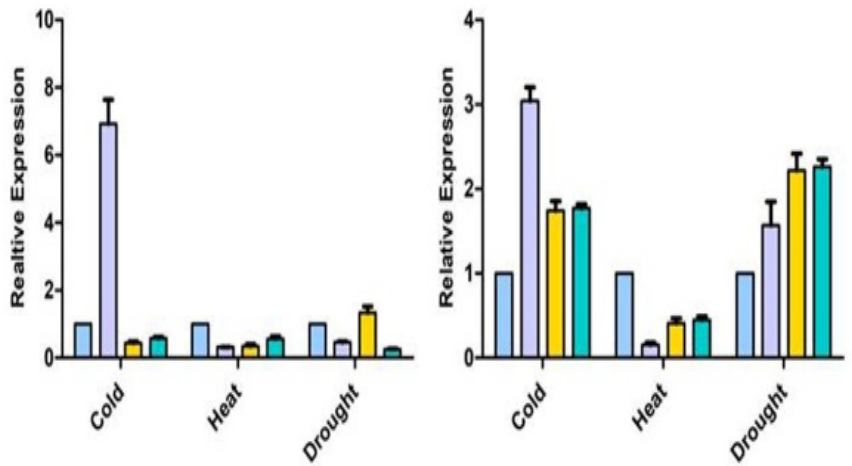

$\mathrm{BB} \times 33$
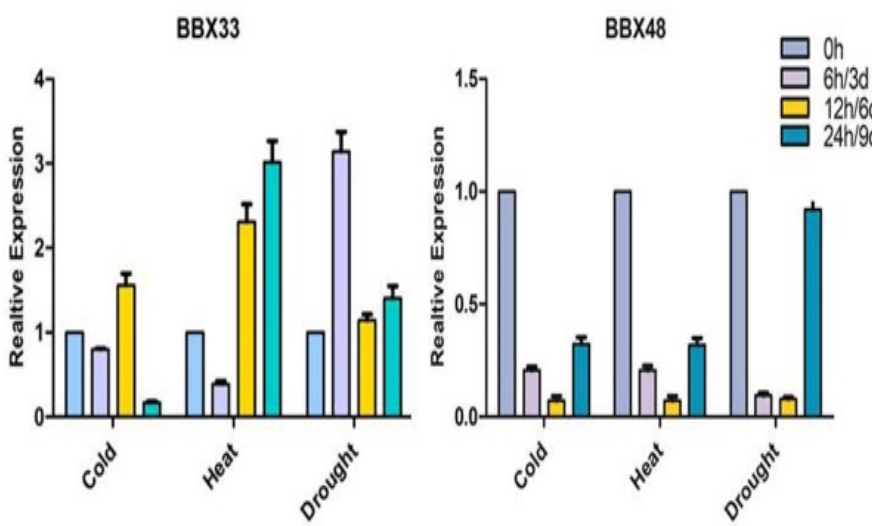

Figure 11. Inducible expression profiles of Brassica BBX genes in response to cold, heat, and drought conditions. The $x$-axis presents the treatments. The $y$-axis presents the expression levels relative to the expression at the 0 -h time point. Error bars indicate the standard deviations of three independent qRT-PCR biological replicates. 
BBX4

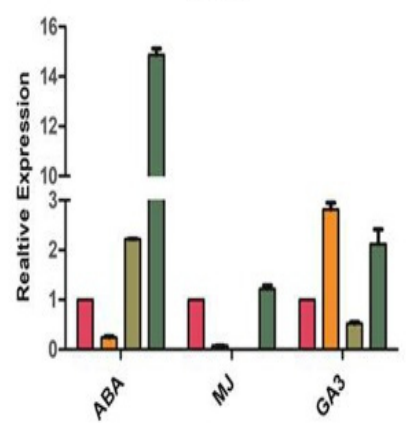

BBX14

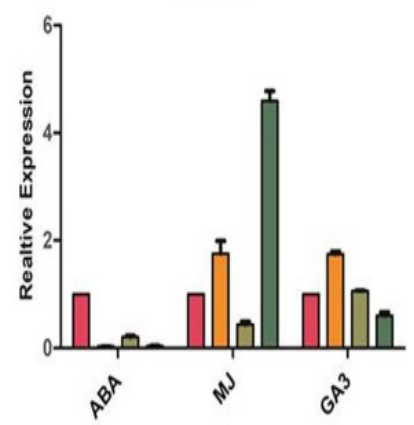

BBX24

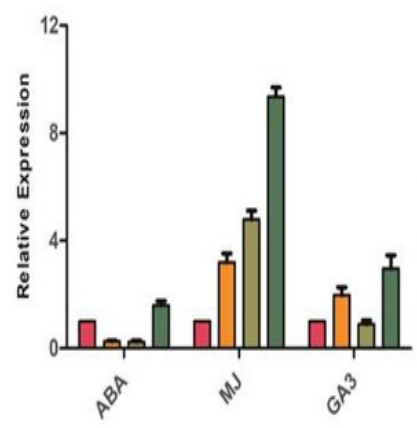

BBX6

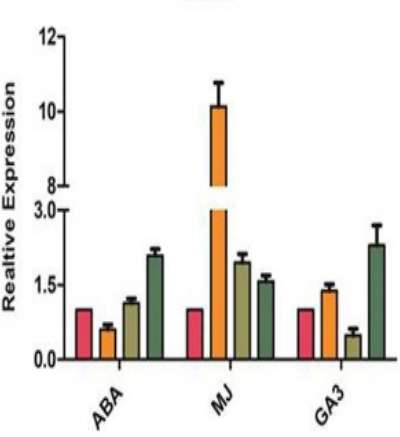

BBX15

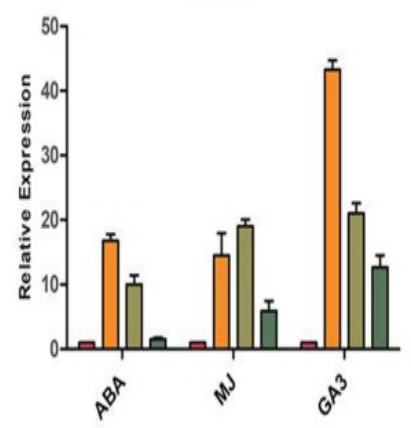

BBX26

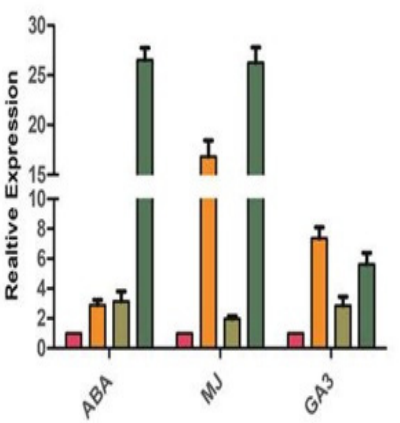

BBX10

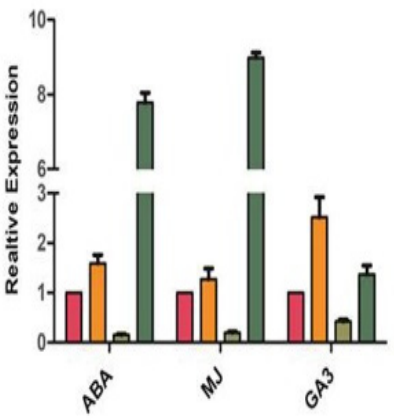

BBX17

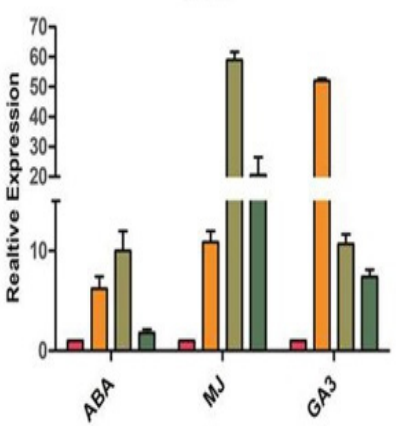

BBX33

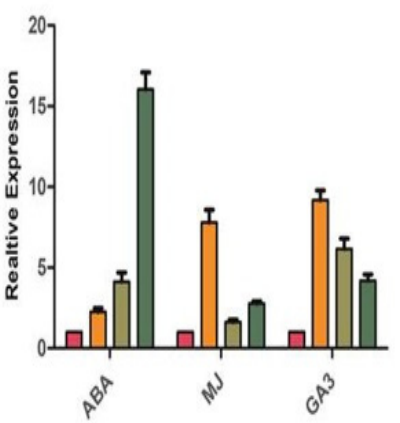

BBX13

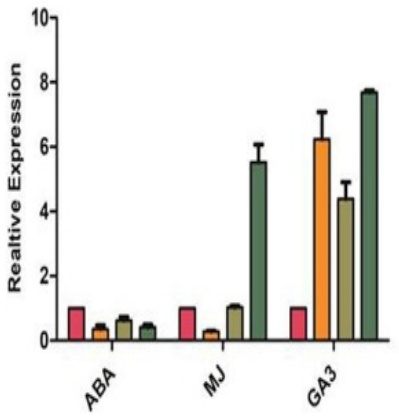

BBX22

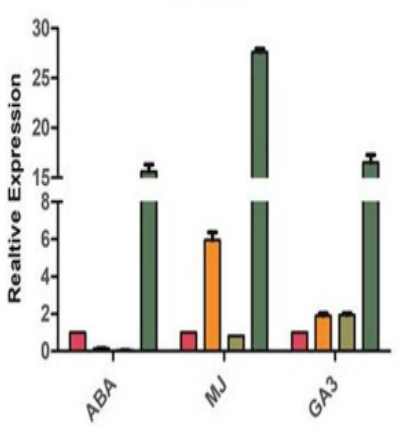

BBX48

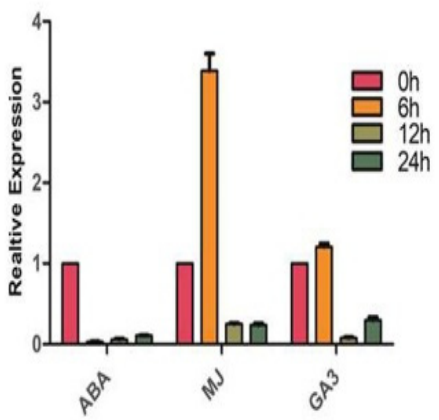

Figure 12. Inducible expression profiles of Brassica BBX genes in response to exogenously applied hormones. The $x$-axis presents the treatments. The $y$-axis presents the expression levels relative to the expression at the 0 -h time point. Error bars indicate the standard deviations of three independent qRT-PCR biological replicates.

We observed that $B r B B X 10, B r B B X 22, B r B B X 26$, and $B r B B X 33$ were highly expressed at some time points following the ABA, MeJA, and GA3 treatments (Figure 12). The BrBBX14, $B r B B X 24$, and $B r B B X 48$ expression levels were upregulated by the application of exogenous MeJA, but were relatively unaffected by ABA and GA3. Interestingly, among the 12 selected $B r B B X$ genes, only $B r B B X 4$ was expressed at very low levels after the exogenous MeJA treatment. Regarding the effects of the exogenous ABA treatment, $B r B B X 15, B r B B X 17$, $B r B B X 26$, and $B r B B X 33$ expression levels were upregulated (minimum 0.5-fold increase) at all time points. Moreover, $B r B B X 6, B r B B X 15, B r B B X 17, B r B B X 24$, and $B r B B X 26$ were also highly expressed at all time points after the MeJA treatment. For both ABA and MeJA, the $B r B B X$ genes were most highly expressed at the 24-h time point. Regarding the effects of GA3, BrBBX13, BrBBX15, BrBBX17, BrBBX22, BrBBX26, and BrBBX33 expression levels were upregulated at almost all time points, but especially at $6 \mathrm{~h}$ post-treatment (Figure 12). 


\section{Discussion}

$B B X$ proteins have recently been confirmed as important transcription factors with vital regulatory roles affecting the economic value of Brassica species, which are important oilseed and vegetable crops worldwide; thus, the $B B X$ gene families in $B$. rapa, $B$. napus, and B. oleracea should be comprehensively investigated. A few Brassica species are susceptible to numerous abiotic factors (e.g., drought, salinity, and temperature extremes) and biotic factors (e.g., bacteria, fungi, insect pests, and viruses) [41]. In the present study, we identified BBX genes in three Brassica species and investigated their phylogenetic relationships, linkage group organization, intron-exon organization, duplication events, conserved motifs, cis-acting elements, and expression patterns in different tissues following various abiotic stress treatments, which is summarized in Table S16. The global identification of Brassica BBX genes provides the foundation for advanced functional studies of these genes in Brassicaceae crops.

\subsection{Identification and Evolution of Brassica BBX Genes}

In this study, we identified 51,52, and 101 BBX genes in B. rapa, B. oleracea, and $B$. napus, respectively. The $B B X$ gene families are larger in these three Brassica species than in other plant species, including $A$. thaliana (32 BBX genes) [4], rice (30 BBX genes) [9], pear (25 $B B X$ genes) [45], tomato (29 $B B X$ genes) [31], potato (30 $B B X$ genes) [47], apple (64 $B B X$ genes) [32], and sweet cherry (15 BBX genes) [52]. To date, the $101 \mathrm{BnBBX}$ genes represent the largest $B B X$ gene family among plant species. These results suggest that the $B B X$ gene families in Brassica species, including B. napus, expanded considerably during speciation. Gene and whole-genome duplication events occurred approximately 35 million years ago in all Brassicaceae genomes $[53,54]$. Moreover, Brassica genomes underwent a lineage-specific whole-genome triplication about 15.9 million years ago $[55,56]$, which may have contributed to the diversification and expansion of the Brassica BBX gene families. Additionally, in the case of $B$. napus, an allopolyploidization followed by the fusion of genomes $A$ and $C$ may have been critical for increasing the number of $B n B B X$ genes [57].

During plant evolution, segmental and tandem duplication events are crucial for expanding gene families [58]. We analyzed the segmental and tandem duplications in Brassica species, which revealed 30, 27, and 64 pairs of BBX genes in B. rapa, B. oleracea, and $B$. napus, respectively, that were derived from segmental duplication events (Tables S5-S7). However, only BrBBX49/BrBBX50 in B. rapa, BoBBX50/BoBBX51 in B. oleracea, and BnBBX92/BnBBX93 in B. napus were identified as tandem duplicates (Table S4). Accordingly, segmental duplications were the major contributor to the expansion of the Brassica $B B X$ families during evolution. The fatty acid desaturase and heat stress transcription factor gene families in Brassica species likely evolved similarly [59,60]. In the current study, the Ka/Ks values of the segmentally duplicated gene pairs were calculated. Usually, $\mathrm{Ka} / \mathrm{Ks}$ values less than and greater than 1 signify negative and positive selection pressures, respectively. A Ka/Ks value equal to 1 reflects neutral selection. The $\mathrm{Ka} / \mathrm{Ks}$ values for the $B B X$ gene pairs were less than 1 in all three Brassica species, with the exception of one gene pair in B. oleracea and B. napus. Hence, most of the Brassica BBX gene pairs evolved under purifying selection. This is consistent with the purifying selection reported for the apple [32] and grapevine [33] BBX gene families. The purifying selection pressure may have helped to maintain the conserved structures of the $B B X$ genes during evolution.

Brassica genomes appear to contain more $B B X$ genes than the genomes of other plant species, including tomato and potato. However, Brassica species have smaller genomes, i.e., $529 \mathrm{Mb}$ in B. rapa [55], $696 \mathrm{Mb}$ in B. oleracea [61], and approximately $1.2 \mathrm{~Gb}$ in B. napus [62], than tomato $(960 \mathrm{Mb})$ [63] and potato $(840 \mathrm{Mb})$ [64]. These findings suggest that the number of $B B X$ genes is not directly related to plant genome size. Moreover, although there were no significant differences in the number of $B B X$ genes among plant species, the $B B X$ gene types varied. For example, the potato genome contains nine, nine, five, and seven $B B X$ genes in Classes I, IV, III and V, respectively [47]. In contrast, the tomato genome includes $8,10,5$, and 6 BBX genes in Classes I, IV, III and V, respectively [31]. In Brassica species, the 
number of genes in these classes is the same in the diploid genomes (i.e., 18, 16, 9, and 9 $B B X$ genes in Classes I, IV, III and V, respectively, in both $B$. rapa and B. oleracea), whereas the allotetraploid amphidiploid genome of $B$. napus contains 34, 20,33, and 14 BBX genes in Classes I, IV, III and V, respectively.

On the basis of the phylogenetic and sequence alignment analyses, the Brassica BBX family members were classified into five subgroups, similar to the BBXs in $A$. thaliana, tomato, pear, and potato [4,31,45-47]. The Brassica BBX family members from Groups I, II, and IV contained the B1 and B2 domains, with the exception of $B r B B X 5, B r B B X 48, B o B B X 4$, and $B o B B X 45$, which should belong to Group III (B1 + CCT), but were in Group II instead $(\mathrm{B} 1+\mathrm{B} 2+\mathrm{CCT})$ (Figure 2). In contrast to animal BBXs with two different BBX domains, the amino acid sequences of the two BBX domains in Brassica species are highly conserved and have the same topology [7]. Most of the BBXs in green algae reportedly only have a single BBX domain, but in the unicellular green alga Chlamydomonas reinhardtii, two BBX domains have been detected in BBXs. These observations imply that $B B X$ gene duplication events occurred in some circumstances well before plants colonized land $[3,6,65]$. During evolution, $B B X$ gene families expanded rapidly. Furthermore, the highly conserved nature of $\mathrm{BBX}$ proteins across the plant kingdom suggests these proteins may have played a significant role in the development of terrestrial plants $[3,65]$.

\subsection{Potential Roles of BrBBX Genes Related to Plant Growth and Development}

Previous studies concluded that $B B X$ genes are important for diverse plant developmental and growth processes, including shade avoidance, seedling photomorphogenesis (e.g., hypocotyl growth), chlorophyll accumulation, and flowering [3,66,67]. In the current study, various cis-acting regulatory elements were identified in the promoters of Brassica $B B X$ genes. Light-responsive cis-regulatory elements were commonly detected in the Brassica BBX gene promoter regions. More specifically, in B. rapa, B. oleracea, and $B$. napus, the following light-responsive elements were identified: ARE, G-box, Box4, GT1motif, AE box, MRE, I-box, chs-CMA, GA motif, GAP-box, 3-AF1 binding site, Sp1, and MSA-like. The circadian cis-element and the GATA cis-element, which are involved in light-regulated and tissue-regulated expression, were identified in the promoter region of 22 B. rapa, 28 B. oleracea, and 43 B. napus $B B X$ genes. These results suggest that the Brassica $B B X$ genes may be functionally similar to $A$. thaliana $A t B B X$ genes, which are under the control of the circadian rhythm and are mostly involved in light-related processes, including flowering, photomorphogenesis, and shade avoidance [3]. In A. thaliana, the overexpression of $A t B B X 6$ (COL5) induces early flowering by upregulating FT expression, whereas the overexpression of $A t B B X 7$ (COL9) delays flowering under short-day conditions because of the associated repressed expression of $F T$ and $C O[15,68]$. Previous research indicated that $A t B B X 32 /$ EIP6 in A. thaliana regulates the flowering time apparently in a $\mathrm{CO}$-independent manner under long-day conditions [69]. In the current study, $B r B B X 6$ and $B r B B X 10$, which are, respectively, homologs of $A t B B X 7$ and $A t B B X 6$, were highly expressed in all examined tissues, except for the silique in the case of $B r B B X 10$. Both genes were expressed at high levels in floral tissues, indicative of a potential regulatory role related to flower development. Additionally, AtBBX21 (i.e., SALT TOLERANCE HOMOLOG 2) positively regulates seedling photomorphogenesis after interacting with HY5, which is a key regulator of photomorphogenesis [66]. Thus, AtBBX21 helps control seed germination and ABA signaling. Moreover, $A t B B X 21$ is expressed at relatively high levels in germinating seeds and dry seeds [70]. In the present study, $B r B B X 32$ and $B r B B X 34$, which are the closest homologs of $A t B B X 21$, were expressed at lower levels in the silique than in all other analyzed tissues. In contrast to AtBBX21 (STH2), both AtBBX24 (STO) and AtBBX25 (STH) suppress seedling photomorphogenesis [19,71]. We observed that $B r B B X 46$, which is closely related to $A t B B X 24$, was most highly expressed in the silique and flower. Hence, $B r B B X$ s might be crucial for $B$. rapa photomorphogenesis. The $B r B B X 46$ gene was expressed in diverse tissues, including the flower, leaf, root, and stem. Accordingly, BrBBX46 might have diverse regulatory functions influencing plant development and growth. Considered 
together, our results suggest that Brassica BBX family members are involved in floral development, while also contributing to seedling photomorphogenesis. They appear to have multiple significant roles in various biological, growth, and developmental processes.

\subsection{Potential Roles of BrBBX Genes in Response to Abiotic Stresses and Exogenous Hormones}

Environmental conditions (e.g., drought, salinity, cold and heat) affect plant growth and development and limit plant productivity [72,73]. Various stresses modulate plant development and growth and modify transcription in plants by affecting specific proteins and post-translational modifications as well as by altering signaling pathways involving many genes [74]. In this study, we proved that Brassica BBX genes are highly responsive to various simulated stresses and hormones, including drought, mannitol, PEG, salt, cold, heat, $\mathrm{ABA}, \mathrm{GA} 3$ and MeJA, implying $B B X$ genes contribute to multiple stress responses in Brassica species. Earlier studies demonstrated the involvement of plant $B B X$ genes in responses to different abiotic stresses and hormone treatments [33,49]. More specifically, the salt tolerance protein STO (AtBBX24) is activated by salt stress in yeast cells [34]. It also increases the root length of $A$. thaliana plants exposed to salinity stress [35]. Additionally, AtBBX18 negatively regulates photomorphogenesis and thermotolerance in A. thaliana, while also negatively regulating the transcription of heat-responsive genes, such as APX2, DGD1, Hsp70 and Hsp101, thereby decreasing the seed germination and seedling survival rates after heat treatment [39]. In chrysanthemum, CmBBX24 mediates drought and cold tolerance and delays flowering [9]. The constitutive expression of $C m B B X 22$ in transgenic A. thaliana leads to enhanced drought tolerance [75]. The BrBBX14 gene, which is a close homolog of $C m B B X 22$, is highly expressed in Brassica species under drought conditions [75]. Furthermore, various studies confirmed that $B B X$ genes affect hormone signaling pathways. A study on $A$. thaliana determined that $B B X$ gene expression is altered by cyclic ADP-ribose (cADPR) at different temperatures and by ABA treatments [76,77]. In pea, BBXs modulate and regulate the COP/HY5 signaling pathway, with $B B X 18$ also possibly affecting GA pathways [78]. In this study, we detected at least one stress-responsive ciselement (e.g., ARE, ABRE, MBS, TC-rich and LTR) in the promoter region of Brassica BBX genes, suggesting the encoded proteins have significant roles in abiotic stress responses (Table S15). Furthermore, the cold treatment substantially affected the expression of at least six genes (BrBBX10, BrBBX14, BrBBX15, BrBBX17, BrBBX22 and BrBBX24), whereas the heat treatment modified the expression of two genes (BrBBX13 and $B r B B X 17)$ (Figure 11). The TC-rich and LTR cis-elements were detected in the promoter region of these genes. Eight genes (BrBBX6, BrBBX10, BrBBX13, BrBBX14, BrBBX15, BrBBX17, BrBBX22, and $B r B B X 24)$ that were expressed at high levels following the drought, mannitol, and PEG treatments have MBS or ABRE elements in their promoter region, with the exception of $B r B B X 17$. Our data indicate that eight Brassica $B B X$ genes highly expressed in response to salt stress have promoters with ABRE, MBS, MYC, or MYB elements. We observed that salt, osmotic, and cold stresses upregulated the expression of most of the Brassica BBX genes, whereas drought and PEG only moderately increased expression and the heat treatment downregulated expression (Figures 10 and 11). These results indicate that the expression of most of the $B r B B X$ genes was induced or repressed to varying degrees depending on the type of stress. Some genes, such as BrBBX15 and BrBBX17 (Figures 10 and 11), were highly expressed in response to all abiotic stresses, suggesting these genes might contribute to responses to multiple stress signals. The BrBBX6 gene was highly expressed following the $\mathrm{NaCl}$, mannitol, and PEG treatments, but its expression was downregulated by cold and heat stress, implying that $B r B B X 6$ might influence tolerance to only specific abiotic stresses. In this study, Brassica $B B X$ gene expression was affected by the application of different hormones (Figure 12). The BrBBX15, BrBBX17, BrBBX22, BrBBX26, and BrBBX33 expression levels were substantially upregulated by ABA, MeJA, and GA3. Most of the $B r B B X$ genes were responsive to $\mathrm{ABA}, \mathrm{MeJA}$, and GA3 at different time points, but gene expression was affected more by MeJA and GA3 than by ABA. This indicates that these genes might regulate various aspects of the Brassica hormone signaling pathways. Only 
$B r B B X 48$ was repressed or unaffected in all treatments. Additionally, we investigated the already characterized B-Box genes in different plant species and found that most of the genes belonged to Group IV (B-box1 + B-box2) and Group V (B-box1) (Table S18). This indicates that Group IV and V are relatively well characterized compared to the rest of the groups for abiotic and hormonal stress. Similarly, in our study, BrBBX13, BrBBX14, $B r B B X 22$ (all three from Group IV) as well as BrBBX4 and BrBBX24 (Group V) are highly expressed in abiotic (heat, cold, salt, and drought) and hormonal (ABA, MeJA, and GA3) stress.

\section{Materials and Methods}

\subsection{Identification of BBX Family Members in Brassica Species}

To identify and annotate $B B X$ genes in $B$. rapa, previously reported $A$. thaliana BBX protein sequences were downloaded from The Arabidopsis Information Resource (TAIR) database (http:/ / www.arabidopsis.org, accessed on 15 April 2020) and used as queries to screen the B. rapa genome database (http:/ / brassicadb.org, accessed on 15 April 2020, version 1.5) using the BLASTP program (http:/ / brassicadb.cn/\#/BLAST/ and https:/ / plants.ensembl.org/Multi/Tools/Blast, accessed on 15 April 2020), and genes with E value $<1 \times 10^{-10}$ were selected. To verify that the identified genes were from the $B B X$ family, all non-redundant protein sequences were checked using the following databases to ensure they were complete and included the targeted domains: NCBI-CDD (https: / / www.ncbi.nlm.nih.gov/Structure/cdd/wrpsb.cgi, accessed on 15 April 2020), Pfam (http://pfam.xfam.org/, accessed on 15 April 2020), and SMART (http://smart.emblheidelberg.de/, accessed on 15 April 2020). For a comparative analysis of the $B B X$ gene family in Brassicaceae, $B B X$ genes were identified in two other Brassicaceae species, namely B. oleracea (http:/ / plants.ensembl.org/index.html, accessed on 15 April 2020) and B. napus (http:/ / plants.ensembl.org/index.html, accessed on 15 April 2020), using the same process. The chromosomal locations of the coding sequences and the number of encoded amino acids in the B. rapa, B. oleracea, and B. napus genes were determined using publicly available databases (http://brassicadb.org and http://plants.ensembl.org/index.html, accessed on 15 April 2020). The grand average of hydropathy (GRAVY) index, isoelectric point $(\mathrm{pI})$, aliphatic index, molecular weight $(\mathrm{kDa})$, and instability index of the BBX proteins in the three Brassica species were calculated using ExPASy (http:/ / www.expasy.org/tools/, accessed on 15 April 2020). The subcellular locations of Brassica BBX proteins were predicted using the online tool WoLF PSORT (https://wolfpsort.hgc.jp/, accessed on 15 April 2020).

\subsection{Chromosomal Location, Tandem Duplication, and Synteny Analyses}

The chromosomal location of all identified Brassica BBX genes was mapped to B. rapa, B. oleracea, and B. napus chromosomes using Mapchart software (Version 2.1) [79] based on the information available at the Brassica genome database (http:/ / brassicadb.org, accessed on 15 April 2020). The physical location of $B B X$ genes on the chromosomes was used to search the tandem duplication using the McScanX toolkit [80]. The circular map of syntenic analysis in the Brassica species genome was constructed using TBtools software [81].

\subsection{Gene Structure, Motif, Conserved Domain, and Phylogenetic Analyses}

On the basis of their genomic and coding sequences, the exon-intron structures of the $B$. rapa, B. oleracea, and $B$. napus $B B X$ genes were analyzed using the Gene Structure Display Server. To identify the conserved domains and BBX motif, the B. rapa, B. oleracea, and $B$. napus BBX protein sequences were analyzed using the NCBI-CDD online portal (https:/ / www.ncbi.nlm.nih.gov/Structure/cdd/wrpsb.cgi, accessed on 20 April 2020,) and the MEME program (https: / / meme-suite.org/meme/, accessed on 15 April 2020). Multiple BBX protein sequences were aligned using ClustalW (version 2.0) and sequence logos were created using the Web logo platform (https: / / weblogo.berkeley.edu/logo.cgi, accessed on 15 April 2020). An unrooted neighbor-joining phylogenetic tree was constructed using MEGA 7.0 (1000 bootstrap replicates) to clarify the evolutionary relationships among 
236 BBX proteins from B. rapa, B. oleracea, B. napus, and A. thaliana (51 from B. rapa, 52 from B. oleracea, 101 from $B$. napus, and 32 from $A$. thaliana).

\subsection{Prediction of Cis-Element}

To identify the putative cis-acting elements found in Brassica BBX genes, the $1.5 \mathrm{~kb}$ genomic sequence upstream of the initiation codon (ATG) of each gene was subjected to PlantCARE (http:/ / bioinformatics.psb.ugent.be/webtools/plantcare/html, accessed on 15 April 2020) [82].

\subsection{Plant Materials, Growth Conditions, and Stress Treatments}

Brassica rapa inbred line Kenshin seeds were germinated at $24{ }^{\circ} \mathrm{C}$ on moistened filter paper in Petri dishes. Four days later, healthy and uniformly growing plants were transferred to pots (16 cm diameter) and then incubated in a growth chamber at $24{ }^{\circ} \mathrm{C}$ with a 16-h light $\left(800 \mu \mathrm{mol} \mathrm{m} \mathrm{m}^{-2} \mathrm{~s}^{-1}\right) / 8$-h dark cycle and $60-65 \%$ relative humidity. Plants were watered equally daily. The effects of abiotic stress and hormone treatments on $B B X$ gene expression were examined using 21-day-old seedlings. Specifically, seedlings (stem and leaf tissue) were sprayed with a solution comprising 20\% polyethylene glycol 6000 (PEG6000), $200 \mathrm{mM}$ mannitol, $200 \mathrm{mM} \mathrm{NaCl}, 50 \mu \mathrm{M}$ abscisic acid (ABA), $100 \mu \mathrm{M}$ gibberellic acid (GA3), $100 \mu \mathrm{M}$ methyl jasmonate (MeJA), or $200 \mu \mathrm{M}$ salicylic acid (SA) (pH 6.5) [83]. The mock control plants were treated with $\mathrm{ddH}_{2} \mathrm{O}$. To simulate heat and cold stresses, plants were incubated in a growth chamber at $42{ }^{\circ} \mathrm{C}$ and $4{ }^{\circ} \mathrm{C}$, respectively. Samples were collected at 0 , 6,12 , and 24 h post-treatment. To simulate drought stress, watering was stopped at 12 days after germination and plants were collected 3,6, and 9 days later. To minimize the effect of the circadian clock on gene expression, control and treated plants were collected at the same time. For all treatments, three biological replicates of plant samples were collected, immediately frozen in liquid nitrogen, and stored at $-80^{\circ} \mathrm{C}$ until use.

\subsection{RNA Isolation, cDNA Synthesis, and Primer Design}

Total RNA was isolated from the collected plant leaves using the RNeasy Plant Mini Kit (Qiagen, Hilden, Germany). Residual genomic DNA was eliminated via an on-column DNase treatment. The RNA quality and quantity were determined using the NanoDrop spectrophotometer (Thermo Scientific, Waltham, MA, USA) and by $1 \%$ agarose gel electrophoresis. Total RNA $(1 \mu \mathrm{g})$ was used as the template for synthesizing cDNA using the TOPscript RT Dry mix kit (Enzynomics, Daejeon, Korea). Quantitative real-time (qRT) PCR primers were designed by IDT, Inc. (Coralville, IA, USA) and are listed in Table S17. For each primer pair, a reverse transcription PCR was performed and the amplified products were examined by $1.5 \%$ agarose gel electrophoresis to confirm they were the expected size.

\subsection{Quantitative Real-Time PCR Analysis}

The cDNA samples were diluted 5 times using nuclease-free water and then $10 \mathrm{ng}$ cDNA was used for each qRT-PCR analysis. The AccuPower 2X GreenStar qPCR MasterMix (Bioneer, Daejeon, Korea) and the CFX96 Touch Real-Time PCR Detection System (Bio-Rad, Hercules, CA, USA) were used for the qRT-PCR analysis. Each $20-\mu \mathrm{L}$ reaction comprised

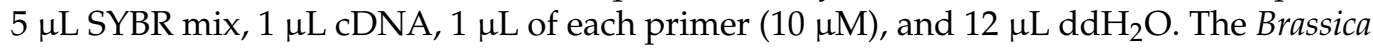
ERF1 gene was used as an internal control for normalizing expression levels (Table S17). The PCR program was as follows: $94{ }^{\circ} \mathrm{C}$ for $5 \mathrm{~min}, 40$ cycles of $94{ }^{\circ} \mathrm{C}$ for $15 \mathrm{~s}, 62{ }^{\circ} \mathrm{C}$ for $20 \mathrm{~s}$, and $72{ }^{\circ} \mathrm{C}$ for $20 \mathrm{~s}$. A melting curve analysis was performed at the end of the qRT-PCR analysis to confirm gene-specific products were amplified. The qRT-PCR was completed using three technical replicates and relative gene expression levels were calculated according to the $2^{-\Delta \Delta C t}$ method [84]. The heat map showed expression patterns of $51 \mathrm{BrBBX}$ genes in different organs and during various developmental stages using available transcriptome data [51]. The relative expression data shown in the heat map were generated using the pheatmap package of $\mathrm{R}$. 


\section{Conclusions}

In this study, we completed a systematic genome-wide analysis of the $B B X$ gene family members in Brassica species. A total of 51,52, and $101 B B X$ genes were identified in B. rapa, B. oleracea, and B. napus, respectively. In all three Brassica species, the genes in the same phylogenetic groups were very similar in terms of their conserved domains and structures. A gene duplication analysis indicated that segmental duplications were primarily responsible for the expansion of the Brassica BBX gene families. Several different cis-acting elements were detected in the promoter regions of the $B B X$ genes in the three analyzed Brassica species, implying these genes are associated with complex regulatory mechanisms and networks governing developmental processes and responses to diverse abiotic stresses and exogenously applied hormones. The observed $B r B B X$ expression patterns in different tissues suggest that $B r B B X$ s may have multiple functions related to the development and growth of Brassica species. Our qRT-PCR analysis of BrBBX expression under various abiotic stress conditions and in response to hormones revealed that $B r B B X s$ may influence abiotic stress tolerance and hormone signaling by modulating multiple stress-responsive and hormone signaling pathways. Overall, the data generated in this study may form the basis of future functional characterizations of Brassica BBX genes, especially regarding plant development and abiotic stress responses.

Supplementary Materials: The following are available online at https:/ /www.mdpi.com/article/10 .3390/ijms221910367/s1. References [85-90] are cited in the Supplementary Materials.

Author Contributions: S.S.C., S.S., S.R.C. and Y.P.L. conceived and designed the study. S.S. and S.S.C. carried out the experiments and generated data. Y.M. and J.J.R. contributed to data analysis and experiments. S.S., S.S.C. and S.R.C. analyzed data. S.S., S.S.C., J.J.R., J.K. and S.H.O. prepared the samples and performed stress treatments. S.S. and S.S.C. drafted the manuscript. S.R.C. and Y.P.L. participated as directors and modified, edited, and finalized the manuscript. All authors have read and agreed to the published version of the manuscript.

Funding: This work was supported by the Korea Institute of Planning and Evaluation for Technology in Food, Agriculture, and Forestry (IPET) through the Golden Seed Project (Grant no. 213006-05-5SBD30, 213006-05-5-SB110), funded by the Ministry of Agriculture, Food and Rural Affairs (MAFRA), the Ministry of Oceans and Fisheries (MOF), the Rural Development Administration (RDA), and Korea Forest Services (KFS).

Institutional Review Board Statement: Not applicable.

Informed Consent Statement: Not applicable.

Data Availability Statement: All the necessary data generated are provided in the form of figures, tables, and supplementary information.

Acknowledgments: The authors appreciate the editors and reviewers for their comments and suggestions to improve this manuscript.

Conflicts of Interest: The authors declare no conflict of interest.

$\begin{array}{ll}\text { Abbreviations } \\ \text { BBX } & \text { B-box } \\ \text { CCT } & \text { CONSTANS, CO-like, and TOC1 } \\ \text { BrBBX } & \text { Brassica rapa B-box } \\ \text { BoBBX } & \text { BoBBX Brassica oleracea B-box } \\ \text { BnBBX } & \text { BnBBX Brassica napus B-box } \\ \text { HMM } & \text { Hidden Markov Model } \\ \text { PEG } & \text { Polyethylene glycol } \\ \text { ABA } & \text { Abscisic acid } \\ \text { GA } & \text { Gibberellic acid } \\ \text { MeJA } & \text { Methyl jasmonate } \\ \text { SA } & \text { Salicylic acid }\end{array}$




\section{References}

1. Vaishak, K.P.; Yadukrishnan, P.; Bakshi, S.; Kushwaha, A.K.; Ramachandran, H.; Job, N.; Babu, D.; Datta, S. The B-box bridge between light and hormones in plants. J. Photochem. Photobiol. B Biol. 2019, 191, 164-174. [CrossRef]

2. Song, Z.; Bian, Y.; Liu, J.; Sun, Y.; Xu, D. B-box proteins: Pivotal players in light-mediated development in plants. J. Integr. Plant Biol. 2020, 62, 1293-1309. [CrossRef]

3. Gangappa, S.N.; Botto, J.F. The BBX family of plant transcription factors. Trends Plant Sci. 2014, 19, 460-470. [CrossRef]

4. Khanna, R.; Kronmiller, B.; Maszle, D.R.; Coupland, G.; Holm, M.; Mizuno, T.; Wu, S.H. The Arabidopsis B-box zinc finger family. Plant Cell 2009, 21, 3416-3420. [CrossRef]

5. Gendron, J.M.; Pruneda-Paz, J.L.; Doherty, C.J.; Gross, A.M.; Kang, S.E.; Kay, S.A. Arabidopsis circadian clock protein, TOC1, is a DNA-binding transcription factor. Proc. Natl. Acad. Sci. USA 2012, 109, 3167-3172. [CrossRef] [PubMed]

6. Crocco, C.D.; Botto, J.F. BBX proteins in green plants: Insights into their evolution, structure, feature and functional diversification. Gene 2013, 531, 44-52. [CrossRef] [PubMed]

7. Strayer, C.; Oyama, T.; Schultz, T.F.; Raman, R.; Somers, D.E.; Más, P.; Panda, S.; Kreps, J.A.; Kay, S.A. Cloning of the Arabidopsis clock gene TOC1, an autoregulatory response regulator homolog. Science 2000, 289, 768-771. [CrossRef]

8. Huang, J.; Zhao, X.; Weng, X.; Wang, L.; Xie, W. The rice B-box zinc finger gene family: Genomic identification, characterization, expression profiling and diurnal analysis. PLoS ONE 2012, 7, e48242. [CrossRef] [PubMed]

9. Yang, Y.; Ma, C.; Xu, Y.; Wei, Q.; Imtiaz, M.; Lan, H.; Gao, S.; Cheng, L.; Wang, M.; Fei, Z.; et al. A zinc finger protein regulates flowering time and abiotic stress tolerance in chrysanthemum by modulating gibberellin biosynthesis. Plant Cell 2014, 26, $2038-2054$. [CrossRef]

10. Putterill, J.; Robson, F.; Lee, K.; Simon, R.; Coupland, G. The CONSTANS gene of Arabidopsis promotes flowering and encodes a protein showing similarities to zinc finger transcription factors. Cell 1995, 80, 847-857. [CrossRef]

11. Talar, U.; Kiełbowicz-Matuk, A. Beyond Arabidopsis: BBX Regulators in Crop Plants. Int. J. Mol. Sci. 2021, 22, 2906. [CrossRef] [PubMed]

12. Samach, A.; Onouchi, H.; Gold, S.E.; Ditta, G.S.; Schwarz-Sommer, Z.; Yanofsky, M.F.; Coupland, G. Distinct roles of CONSTANS target genes in reproductive development of Arabidopsis. Science 2000, 288, 1613-1616. [CrossRef] [PubMed]

13. Suárez-López, P.; Wheatley, K.; Robson, F.; Onouchi, H.; Valverde, F.; Coupland, G. CONSTANS mediates between the circadian clock and the control of flowering in Arabidopsis. Nature 2001, 410, 1116-1120. [CrossRef] [PubMed]

14. Valverde, F.; Mouradov, A.; Soppe, W.; Ravenscroft, D.; Samach, A.; Coupland, G. Photoreceptor regulation of CONSTANS protein in photoperiodic flowering. Science 2004, 303, 1003-1006. [CrossRef] [PubMed]

15. Cheng, X.F.; Wang, Z.Y. Overexpression of COL9, a CONSTANS-LIKE gene, delays flowering by reducing expression of CO and FT in Arabidopsis thaliana. Plant J. 2005, 43, 758-768. [CrossRef]

16. Datta, S.; Hettiarachchi, G.H.C.M.; Deng, X.W.; Holm, M. Arabidopsis CONSTANS-LIKE3 is a positive regulator of red light signaling and root growth. Plant Cell 2006, 18, 70-84. [CrossRef]

17. Tripathi, P.; Carvallo, M.; Hamilton, E.E.; Preuss, S.; Kay, S.A. Arabidopsis B-BOX32 interacts with CONSTANS-LIKE3 to regulate flowering. Proc. Natl. Acad. Sci. USA 2017, 114, 172-177. [CrossRef]

18. Fan, X.Y.; Sun, Y.; Cao, D.M.; Bai, M.Y.; Luo, X.M.; Yang, H.J.; Wei, C.Q.; Zhu, S.W.; Sun, Y.; Chong, K.; et al. BZS1, a B-box protein, promotes photomorphogenesis downstream of both brassinosteroid and light signaling pathways. Mol. Plant 2012, 5, 591-600. [CrossRef]

19. Gangappa, S.N.; Crocco, C.D.; Johansson, H.; Datta, S.; Hettiarachchi, C.; Holm, M.; Botto, J.F. The Arabidopsis B-BOX protein $B B X 25$ interacts with $H Y 5$, negatively regulating BBX22 expression to suppress seedling photomorphogenesis. Plant Cell 2013, 25, 1243-1257. [CrossRef]

20. Lin, F.; Jiang, Y.; Li, J.; Yan, T.; Fan, L.; Liang, J.; Chen, Z.J.; Xu, D.; Deng, X.W. B-BOX DOMAIN PROTEIN28 negatively regulates photomorphogenesis by repressing the activity of transcription factor HY5 and undergoes COP1-mediated degradation. Plant Cell 2018, 30, 2006-2019. [CrossRef]

21. Xu, D.; Jiang, Y.; Li, J.; Lin, F.; Holm, M.; Deng, X.W. BBX21, an Arabidopsis B-box protein, directly activates HY5 and is targeted by COP1 for 26S proteasome-mediated degradation. Proc. Natl. Acad. Sci. USA 2016, 113, 7655-7660. [CrossRef] [PubMed]

22. Xu, D.; Jiang, Y.; Li, J.; Holm, M.; Deng, X.W. The B-box domain protein BBX21 promotes photomorphogenesis. Plant Physiol. 2018, 176, 2365-2375. [CrossRef] [PubMed]

23. Zhang, X.; Huai, J.; Shang, F.; Xu, G.; Tang, W.; Jing, Y.; Lin, R. A PIF1/PIF3-HY5-BBX23 transcription factor cascade affects photomorphogenesis. Plant Physiol. 2017, 174, 2487-2500. [CrossRef]

24. Xu, D. COP1 and BBXs-HY5-mediated light signal transduction in plants. New Phytol. 2019, 228, 1748-1753. [CrossRef]

25. Zhao, X.; Heng, Y.; Wang, X.; Deng, X.W.; Xu, D. A positive feedback loop of BBX11-BBX21-HY5 promotes photomorphogenic development in Arabidopsis. Plant Commun. 2020, 1, 100045. [CrossRef]

26. Heng, Y.; Jiang, Y.; Zhao, X.; Zhou, H.; Wang, X.; Deng, X.W.; Xu, D. BBX4, a phyB-interacting and modulated regulator, directly interacts with PIF3 to fine tune red light-mediated photomorphogenesis. Proc. Natl. Acad. Sci. USA 2019, 116, 26049-26056. [CrossRef] [PubMed]

27. An, J.P.; Wang, X.F.; Zhang, X.W.; Bi, S.Q.; You, C.X.; Hao, Y.J. MdBBX22 regulates UV-B-induced anthocyanin biosynthesis through regulating the function of MdHY5 and is targeted by MdBT2 for 26S proteasome-mediated degradation. Plant Biotechnol. 2019, 17, 2231-2233. [CrossRef] 
28. An, J.P.; Wang, X.F.; Espley, R.V.; Lin-Wang, K.; Bi, S.Q.; You, C.X.; Hao, Y.J. An apple B-Box protein MdBBX37 modulates anthocyanin biosynthesis and hypocotyl elongation synergistically with MdMYBs and MdHY5. Plant Cell Physiol. 2020, 61, 130-143. [CrossRef]

29. Bai, B.; Lu, N.; Li, Y.; Guo, S.; Yin, H.; He, Y.; Sun, W.; Li, W.; Xie, X. OsBBX14 promotes photomorphogenesis in rice by activating OsHY5L1 expression under blue light conditions. Plant Sci. 2019, 84, 192-202. [CrossRef]

30. Xiong, C.; Luo, D.; Lin, A.; Zhang, C.; Shan, L.; He, P.; Li, B.; Zhang, Q.; Hua, B.; Yuan, Z.; et al. A tomato B-box protein Sl BBX 20 modulates carotenoid biosynthesis by directly activating PHYTOENE SYNTHASE 1, and is targeted for 26S proteasome-mediated degradation. New Phytol. 2019, 221, 279-294. [CrossRef]

31. Chu, Z.; Wang, X.; Li, Y.; Yu, H.; Li, J.; Lu, Y.; Li, H.; Ouyang, B. Genomic organization, phylogenetic and expression analysis of the B-BOX gene family in tomato. Front. Plant Sci. 2016, 7, 1552. [CrossRef]

32. Liu, X.; Li, R.; Dai, Y.; Chen, X.; Wang, X. Genome-wide identification and expression analysis of the B-box gene family in the Apple (Malus domestica Borkh.) genome. Mol. Genet. Genom. 2018, 293, 303-315. [CrossRef]

33. Wei, H.; Wang, P.; Chen, J.; Li, C.; Wang, Y.; Yuan, Y.; Fang, J.; Leng, X. Genome-wide identification and analysis of B-BOX gene family in grapevine reveal its potential functions in berry development. BMC Plant Biol. 2020, 20, 1-19. [CrossRef]

34. Lippuner, V.; Cyert, M.S.; Gasser, C.S. Two classes of plant cDNA clones differentially complement yeast calcineurin mutants and increase salt tolerance of wild-type yeast. J. Biol. Chem. 1996, 271, 12859-12866. [CrossRef]

35. Nagaoka, S.; Takano, T. Salt tolerance-related protein STO binds to a Myb transcription factor homologue and confers salt tolerance in Arabidopsis. J. Exp. Bot. 2003, 54, 2231-2237. [CrossRef]

36. Belles-Boix, E.; Babiychuk, E.; Van Montagu, M.; Inzé, D.; Kushnir, S. CEO1, a new protein from Arabidopsis thaliana, protects yeast against oxidative damage. FEBS Lett. 2000, 482, 19-24. [CrossRef]

37. Fujibe, T.; Saji, H.; Arakawa, K.; Yabe, N.; Takeuchi, Y.; Yamamoto, K.T. A methyl viologen-resistant mutant of Arabidopsis, which is allelic to ozone-sensitive $r c d 1$, is tolerant to supplemental ultraviolet-B irradiation. Plant Physiol. 2004, 134, 275-285. [CrossRef] [PubMed]

38. Kobayashi, M.; Horiuchi, H.; Fujita, K.; Takuhara, Y.; Suzuki, S. Characterization of grape C-repeat-binding factor 2 and B-boxtype zinc finger protein in transgenic Arabidopsis plants under stress conditions. Mol. Biol. Rep. 2012, 39, 7933-7939. [CrossRef] [PubMed]

39. Wang, Q.; Tu, X.; Zhang, J.; Chen, X.; Rao, L. Heat stress-induced BBX18 negatively regulates the thermotolerance in Arabidopsis. Mol. Biol. Rep. 2013, 40, 2679-2688. [CrossRef] [PubMed]

40. Wang, Q.; Zeng, J.; Deng, K.; Tu, X.; Zhao, X.; Tang, D.; Liu, X. DBB1a, involved in gibberellin homeostasis, functions as a negative regulator of blue light-mediated hypocotyl elongation in Arabidopsis. Planta 2011, 233, 13-23. [CrossRef]

41. Kayum, M.A.; Kim, H.T.; Nath, U.K.; Park, J.I.; Kho, K.H.; Cho, Y.G.; Nou, I.S. Research on biotic and abiotic stress related genes exploration and prediction in Brassica rapa and B. oleracea: A review. Plant Breed. Biotechnol. 2016, 4, 135-144. [CrossRef]

42. Lee, B.R.; Zaman, R.; Avice, J.C.; Ourry, A.; Kim, T.H. Sulfur use efficiency is a significant determinant of drought stress tolerance in relation to photosynthetic activity in Brassica napus cultivars. Front. Plant Sci. 2016, 7, 459. [CrossRef] [PubMed]

43. Verma, S.K.; Das, A.K.; Gantait, S.; Kumar, V.; Gurel, E. Applications of carbon nanomaterials in the plant system: A perspective view on the pros and cons. Sci. Total Environ. 2019, 667, 485-499. [CrossRef] [PubMed]

44. Wu, F.; Price, B.W.; Haider, W.; Seufferheld, G.; Nelson, R.; Hanzawa, Y. Functional and evolutionary characterization of the CONSTANS gene family in short-day photoperiodic flowering in soybean. PLoS ONE 2014, 9, e85754.

45. Cao, Y.; Han, Y.; Meng, D.; Li, D.; Jiao, C.; Jin, Q.; Lin, Y.; Cai, Y. B-BOX genes: Genome-wide identification, evolution and their contribution to pollen growth in pear (Pyrus bretschneideri Rehd.). BMC Plant Biol. 2017, 17, 156. [CrossRef] [PubMed]

46. Zou, Z.; Wang, R.; Wang, R.; Yang, S.; Yang, Y. Genome-wide identification, phylogenetic analysis, and expression profiling of the BBX family genes in pear. J. Hortic. Sci. Biotechnol. 2018, 93, 37-50. [CrossRef]

47. Talar, U.; Kiełbowicz-Matuk, A.; Czarnecka, J.; Rorat, T. Genome-wide survey of B-box proteins in potato (Solanum tuberosum) Identification, characterization and expression patterns during diurnal cycle, etiolation and de-etiolation. PLoS ONE 2017, 12, e0177471. [CrossRef]

48. Shalmani, A.; Fan, S.; Jia, P.; Li, G.; Muhammad, I.; Li, Y.; Sharif, R.; Dong, F.; Zuo, X.; Li, K.; et al. Genome Identification of B-BOX Gene Family Members in Seven Rosaceae Species and Their Expression Analysis in Response to Flower Induction in Malus domestica. Molecules 2018, 23, 1763. [CrossRef]

49. Shalmani, A.; Jing, X.Q.; Shi, Y.; Muhammad, I.; Zhou, M.R.; Wei, X.Y.; Chen, Q.Q.; Li, W.Q.; Liu, W.T.; Chen, K.M. Characterization of B-BOX gene family and their expression profiles under hormonal, abiotic and metal stresses in Poaceae plants. BMC Genom. 2019, 20, 27. [CrossRef]

50. Voorrips, R.E. MapChart: Software for the graphical presentation of linkage maps and QTLs. J. Hered. 2002, 93, 77-78. [CrossRef]

51. Wang, Y.; Tang, H.; DeBarry, J.D.; Tan, X.; Li, J.; Wang, X.; Lee, T.H.; Jin, H.; Marler, B.; Guo, H.; et al. MCScanX: A toolkit for detection and evolutionary analysis of gene synteny and collinearity. Nucleic Acids Res. 2012, 40, e49. [CrossRef]

52. Chen, C.; Chen, H.; Zhang, Y.; Thomas, H.R.; Frank, M.H.; He, Y.; Xia, R. TBtools-an integrative toolkit developed for interactive analyses of big biological data. Mol. Plant 2020, 13, 1194-1202. [CrossRef]

53. Lescot, M.; Déhais, P.; Thijs, G.; Marchal, K.; Moreau, Y.; Van de Peer, Y.; Rouzé, P.; Rombauts, S. PlantCARE, a database of plant cis-acting regulatory elements and a portal to tools for in silico analysis of promoter sequences. Nucleic Acids Res. 2002, 30, 325-327. [CrossRef] [PubMed] 
54. Saha, G.; Park, J.I.; Ahmed, N.U.; Kayum, M.A.; Kang, K.K.; Nou, I.S. Characterization and expression profiling of MYB transcription factors against stresses and during male organ development in Chinese cabbage (Brassica rapa ssp. pekinensis). Plant Physiol. Biochem. 2016, 104, 200-215. [CrossRef]

55. Livak, K.J.; Schmittgen, T.D. Analysis of relative gene expression data using real-time quantitative PCR and the $2^{-\Delta \Delta C T}$ method. Methods 2001, 25, 402-408. [CrossRef] [PubMed]

56. Yoon, Y.; Seo, D.H.; Shin, H.; Kim, H.J.; Kim, C.M.; Jang, G. The Role of stress-responsive transcription factors in modulating abiotic stress tolerance in plants. Agron 2020, 10, 788. [CrossRef]

57. Tong, C.; Wang, X.; Yu, J.; Wu, J.; Li, W.; Huang, J.; Dong, C.; Hua, W.; Liu, S. Comprehensive analysis of RNA-seq data reveals the complexity of the transcriptome in Brassica rapa. BMC Genom. 2013, 14, 1-10. [CrossRef]

58. Wang, Y.; Zhai, Z.; Sun, Y.; Feng, C.; Peng, X.; Zhang, X.; Xiao, Y.; Zhou, X.; Wang, W.; Jiao, J.; et al. Genome-Wide Identification of the B-BOX Genes that Respond to Multiple Ripening Related Signals in Sweet Cherry Fruit. Int. J. Mol. Sci. 2021, $22,1622$. [CrossRef]

59. Bowers, J.E.; Chapman, B.A.; Rong, J.; Paterson, A.H. Unravelling angiosperm genome evolution by phylogenetic analysis of chromosomal duplication events. Nature 2003, 422, 433-438. [CrossRef] [PubMed]

60. Jiao, Y.; Wickett, N.J.; Ayyampalayam, S.; Chanderbali, A.S.; Landherr, L.; Ralph, P.E.; Tomsho, L.P. A genome triplication associated with early diversification of the core eudicots. Genome Biol. 2012, 13, 1-14. [CrossRef]

61. Wang, X.; Wang, H.; Wang, J.; Sun, R.; Wu, J.; Liu, S.; Bai, Y.; Mun, J.H.; Bancroft, I.; Cheng, F.; et al. The genome of the mesopolyploid crop species Brassica rapa. Nat. Genet. 2011, 43, 1035-1039. [CrossRef]

62. Lysak, M.A.; Koch, M.A.; Pecinka, A.; Schubert, I. Chromosome triplication found across the tribe Brassiceae. Genome Res. 2005, 15, 516-525. [CrossRef]

63. Cheng, F.; Wu, J.; Wang, X. Genome triplication drove the diversification of Brassica plants. Hortic. Res. 2014, 1, 1-8. [CrossRef]

64. Cannon, S.B.; Mitra, A.; Baumgarten, A.; Young, N.D.; May, G. The roles of segmental and tandem gene duplication in the evolution of large gene families in Arabidopsis thaliana. BMC Plant Biol. 2004, 4, 10. [CrossRef] [PubMed]

65. Xue, Y.; Chen, B.; Wang, R.; Win, A.N.; Li, J.; Chai, Y. Genome-wide survey and characterization of fatty acid desaturase gene family in Brassica napus and its parental species. Appl. Biochem. Biotechnol. 2018, 184, 582-598. [CrossRef] [PubMed]

66. Lohani, N.; Golicz, A.A.; Singh, M.B.; Bhalla, P.L. Genome-wide analysis of the Hsf gene family in Brassica oleracea and a comparative analysis of the Hsf gene family in B. oleracea, B. rapa and B. napus. Funct. Integr. Genom. 2019, 19, 515-531. [CrossRef] [PubMed]

67. Liu, S.; Liu, Y.; Yang, X.; Tong, C.; Edwards, D.; Parkin, I.A.; Zhao, M.; Ma, J.; Yu, J.; Huang, S.; et al. The Brassica oleracea genome reveals the asymmetrical evolution of polyploid genomes. Nat. Commun. 2014, 5, 1-11. [CrossRef] [PubMed]

68. Song, J.M.; Guan, Z.; Hu, J.; Guo, C.; Yang, Z.; Wang, S.; Liu, D.; Wang, B.; Lu, S.; Zhou, R.; et al. Eight high-quality genomes reveal pan-genome architecture and ecotype differentiation of Brassica napus. Nat. Plants 2020, 6, 34-45. [CrossRef] [PubMed]

69. Tomato Genome Consortium. The tomato genome sequence provides insights into fleshy fruit evolution. Nature 2012, $485,635$. [CrossRef]

70. Potato Genome Sequencing Consortium. Genome sequence and analysis of the tuber crop potato. Nature 2011, 475, 189. [CrossRef]

71. Kenrick, P.; Crane, P.R. The origin and early evolution of plants on land. Nature 1997, 389, 33-39. [CrossRef]

72. Datta, S.; Hettiarachchi, C.; Johansson, H.; Holm, M. Salt Tolerance HOMOLOG2, a B-Box protein in Arabidopsis that activates transcription and positively regulates light-mediated development. Plant Cell 2007, 19, 3242-3255. [CrossRef]

73. Datta, S.; Johansson, H.; Hettiarachchi, C.; Irigoyen, M.L.; Desai, M.; Rubio, V.; Holm, M. LZF1/Salt Tolerance HOMOLOG3, an Arabidopsis B-box protein involved in light- dependent development and gene expression, undergoes COP1-mediated ubiquitination. Plant Cell 2008, 20, 2324-2338. [CrossRef]

74. Hassidim, M.; Harir, Y.; Yakir, E.; Kron, I.; Green, R.M. Over-expression of CONSTANS-LIKE 5 can induce flowering in short-day grown Arabidopsis. Planta 2009, 230, 481-491. [CrossRef]

75. Park, H.Y.; Lee, S.Y.; Seok, H.Y.; Kim, S.H.; Sung, Z.R.; Moon, Y.H. EMF1 interacts with EIP1, EIP6 or EIP9 involved in the regulation of flowering time in Arabidopsis. Plant Cell Physiol. 2011, 52, 1376-1388. [CrossRef]

76. Xu, D.; Li, J.; Gangappa, S.N.; Hettiarachchi, C.; Lin, F.; Andersson, M.X.; Jiang, Y.; Deng, X.W.; Holm, M. Convergence of light and ABA signaling on the ABI5 promoter. PLoS Genet. 2014, 10, e1004197. [CrossRef]

77. Gangappa, S.N.; Holm, M.; Botto, J.F. Molecular interactions of BBX24 and BBX25 with HYH, HY5 HOMOLOG, to modulate Arabidopsis seedling development. Plant Signal. Behav. 2013, 8, e25208. [CrossRef] [PubMed]

78. You, J.; Chan, Z. ROS regulation during abiotic stress responses in crop plants. Front. Plant Sci. 2015, 6, 1092. [CrossRef] [PubMed]

79. Zhu, J.K. Abiotic stress signaling and responses in plants. Cell 2016, 167, 313-324. [CrossRef] [PubMed]

80. Bechtold, U.; Field, B. Molecular mechanisms controlling plant growth during abiotic stress. J. Exp. Bot. 2018, 69, 2753-2758. [CrossRef]

81. Liu, Y.; Chen, H.; Ping, Q.; Zhang, Z.; Guan, Z.; Fang, W.; Chen, S.; Chen, F.; Jiang, J.; Zhang, F. The heterologous expression of CmBBX22 delays leaf senescence and improves drought tolerance in Arabidopsis. Plant Cell Rep. 2019, 38, 15-24. [CrossRef]

82. Sánchez, J.P.; Duque, P.; Chua, N.H. ABA activates ADPR cyclase and cADPR induces a subset of ABA-responsive genes in Arabidopsis. Plant J. 2004, 38, 381-395. [CrossRef]

83. Soitamo, A.J.; Piippo, M.; Allahverdiyeva, Y.; Battchikova, N.; Aro, E.M. Light has a specific role in modulating Arabidopsis gene expression at low temperature. BMC Plant Biol. 2008, 8, 1-20. [CrossRef] 
84. Weller, J.L.; Hecht, V.; Vander Schoor, J.K.; Davidson, S.E.; Ross, J.J. Light regulation of gibberellin biosynthesis in pea is mediated through the COP1/HY5 pathway. Plant Cell 2019, 21, 800-813. [CrossRef]

85. Takuhara, Y.; Kobayashi, M.; Suzuki, S. Low-temperature-induced transcription factors in grapevine enhance cold tolerance in transgenic Arabidopsis plants. J. Plant Physiol. 2011, 168, 967-975. [CrossRef] [PubMed]

86. Bai, S.; Tao, R.; Tang, Y.; Yin, L.; Ma, Y.; Ni, J.; Yan, X.; Yang, Q.; Wu, Z.; Zeng, Y.; et al. BBX16, a B-box protein, positively regulates light-induced anthocyanin accumulation by activating MYB10 in red pear. Plant Biotechnol. J. 2019, 17, 1985-1997. [CrossRef] [PubMed]

87. Bai, S.; Tao, R.; Yin, L.; Ni, J.; Yang, Q.; Yan, X.; Yang, F.; Guo, X.; Li, H.; Teng, Y. Two B-box proteins, PpBBX18 and PpBBX21, antagonistically regulate anthocyanin biosynthesis via competitive association with Pyrus pyrifolia ELONGATED HYPOCOTYL 5 in the peel of pear fruit. Plant J. 2019, 100, 1208-1223. [CrossRef] [PubMed]

88. Liu, X.; Li, R.; Dai, Y.; Yuan, L.; Sun, Q.; Zhang, S.; Wang, X. A B-box zinc finger protein, Md BBX10, enhanced salt and drought stresses tolerance in Arabidopsis. Plant Mol. Biol. 2019, 99, 437-447. [CrossRef]

89. Mbambalala, N.; Panda, S.K.; van der Vyver, C. Overexpression of AtBBX29 improves drought tolerance by maintaining photosynthesis and enhancing the antioxidant and osmolyte capacity of sugarcane plants. Plant Mol. Biol. Rep. 2021, 39, 419-433. [CrossRef]

90. Wang, M.J.; Ding, L.; Liu, X.H.; Liu, J.X. Two B-box domain proteins, BBX28 and BBX29, regulate flowering time at low ambient temperature in Arabidopsis. Plant Mol. Biol. 2021, 106, 21-32. [CrossRef] 